NASA TECHNICAL NOTE

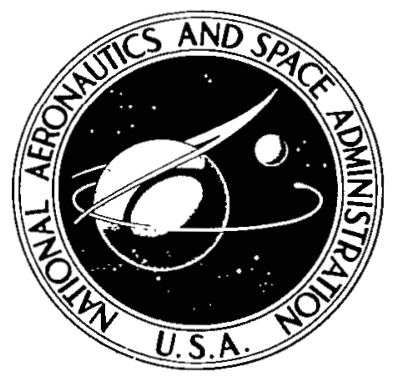

NASA IN D-5451 C. 1

5
5
$\vdots$
$z$
$\vdots$
$\Sigma$

LOAN COPY : RETURN TO AFWL (WLOL)

KIRTLAND AFB, N MEX

\title{
A MINIATURE ELECTROMECHANICAL TUNNEL DIODE TRANSDUCER
}

by W. Rindner, A. Garfein, E. Pittelli, and A. Iannini

Electronics Research Center

Cambridge, Mass.

NATIONAL AERONAUtics AND SPACE ADMINISTRATION - WASHINGTON, D. C. - NOVEMBER 1969 
1. Report No. NASA TN D-5451

2. Government Accossion No.

4. Title and Subtitlo

A Miniature Electromechanical

Tunnel Diode Transducer

7. Author(s) W. Rindner, A. Garfein,

E. Pittelil, and Á. Iannini

9. Performing Organization Name and Address

Electronics Research Center

Cambridge, Mass.

12. Sponsoring Agency Name and Address

National Aeronautics and

Space Administration

Washington, D.C. 20546

15. Supplementary Notes

16. Abstract

The theory, fabrication, and performance of new gallium antimonide tunnel diode transducers are described. The outstanding features of these devices are simplicity, very small size (< $1 \mathrm{~mm})$, good resolution ( $0.3 \mathrm{~mm} \mathrm{Hg}$ for a $1000 \mathrm{~Hz}$ bandwidth) and wide dynamic range ( $260 \mathrm{~dB}$ ) The temperature sensitivity is generally, but not inherently, high (equivalent to $\sim 25 \mathrm{~mm} \mathrm{Hg} /{ }^{\circ} \mathrm{C}$ ) but is readily reduced by conventional compensation techniques to the equivalent of about $1 \mathrm{~mm} \mathrm{Hg} /{ }^{\circ} \mathrm{C}$ or better. The present design is particularly attractive for biomedical applications for which special configurations and circuitry have been developed. In addition, an experimental tunnel diode accelerometer of miniature size and mass is described. Taking advantage of the tunnel diode switching characteristic, experimental circuitry has been designed to yield pulse-width and frequency-modulated output with high percentage ( $\sim 0.5$ percent $/ \mathrm{mm} \mathrm{Hg})$ deviation.

17. Key Words

- Electromechanical

Transducer

- Tunnel Diode

- Biomedical Applications

19. Security Clossif. (of this report) Unclassified

20. Security Classif. (of this page) Unclassified

* For sale by the Clearinghouse for Federal Scientific and Technical Information Springfield, Virginia 22151

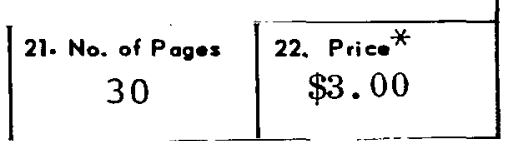


A MINIATURE ELECTROMECHANICAL TUNNEL DIODE TRANSDUCER

By Wilhelm Rindner, Andre Garfein, Ernest Pittelli, and Americo Iannini

Electronics Research Center

Cambridge, Massachusetts

\section{SUMMARY}

The theory, fabrication, and performance of new gallium antimonide tunnel diode transducers are described. The outstanding features of these devices are simplicity, very small size (< $1 \mathrm{~mm})$, good resolution ( $0.3 \mathrm{~mm} \mathrm{Hg}$ for a $1000 \mathrm{~Hz}$ bandwidth) and wide dynamic range ( $60 \mathrm{db})$. The temperature sensitivity is generally, but not inherently, high (equivalent to $\sim 25 \mathrm{~mm} \mathrm{Hg} /{ }^{\circ} \mathrm{C}$ ) but is readily reduced by conventional compensation techniques to the equivalent of about $1 \mathrm{~mm} \mathrm{Hg} /{ }^{\circ} \mathrm{C}$ or better. The present design is particularly attractive for biomedical applications for which special configurations and circuitry have been developed. In addition, an experimental tunnel diode accelerometer of miniature size and mass is described. Experimental circuitry based on the tunnel diode switching characteristic has been designed to yield pulse-width and frequency-modulated output with high percentage ( 0.5 percent/mm $\mathrm{Hg}$ ) deviation.

\section{INTRODUCTION}

Very shortly following Esaki's already classic paper (ref. 1) in 1958, it became evident that the electrical characteristics of degenerate semiconductor tunnel diodes are sensitive to mechanical pressure (ref. 2). Since then, various efforts have been undertaken to utilize this effect in new electromechanical transducers (refs. 3, 4, 5). Such devices, although subject to limitaiions in linearity and bias requirements, were expected to have very attractive properties: in particular, high sensitivity combined with very small dimensions, and temperature insensitivity. However, it appears that until now, a decade later, the tunnel diode transducer still has not gone beyond the laboratory stage.

Our own efforts have led us to believe that one of the difficulties which were experienced in the practical realization of the tunnel diode transducer (TDT) was related to the instabilities and noise caused by unrecognized mechanical damage to the junction. Junctions under true hydrostatic pressure do not suffer from this problem; however, their usefulness is limited in practice to very high pressure ranges. We have, therefore, aimed our efforts at the development of simple fabrication techniques that safeguard the mechanical integrity of the junction while subjecting 
it to anisotropic stress. At the same time, we have concentrated on the use of Gasb junctions, which have larger pressure sensitivity than Ge, the principal material previously used. The resulting new device has proven its usefulness in the biomedical research field, and with further development a broader range of applications appears well within reach. Since the TDT cannot readily be used in conjunction with conventional transducer circuitry, we have also extended our efforts to include some circuit development.

In order to present a reasonably complete picture, we include a brief analysis of the physical mechanisms on which the devices are based and discuss the large excess current effects whose presence appears incompatible with predictable and stable device operation. These effects are attributed to damage-induced defect levels, the dynamics of which are now being studied using the TDT as a convenient research tool.

\section{DEVICE THEORY}

In the following, we shall briefly review the theory of the tunnel diode, confining ourselves to those aspects which are pertinent to our transducer application.

It will be recalled that the tunnel diode incorporates a highIy abrupt $\mathrm{p}-\mathrm{n}$ junction which is so heavily doped that the Fermi level lies in the valence and conduction bands of the $p$ and $n$ sides, respectively (Figure 1). Forward bias causes electrons in the conduction band on the n-type side to tunnel through the junction into empty states of the same energy in the valence band on the p-side. This tunnel current reaches a maximum $I_{p}$ at a bias $V_{p}$ which raises a maximum number of electrons to energies of valence band holes. An increase in bias above $V_{p}$ reduces the current by reducing band overlap until, at a sufticiently high bias, the bottom of the conduction band on the n-side rises above the top of the p-side valence band, and the tunneling current vanishes. The excess current $I_{X}$ beyond this bias comes about by indirect tunneling via levels in the band gap believed to be associated primarily with defects. An increase of bias substantially beyond the valley gives rise predominantly to diffusion current. Pressure effects on diffusion currents in diodes and transistors have been amply covered before and need not concern us here (refs. $8,9)$.

The primary mode of operation of the TDT is based on variation of the peak current caused by a pressure-induced change in the band gap $\mathrm{E}_{\mathrm{G}}$. At the peak voltage the current is (ref. 10): 


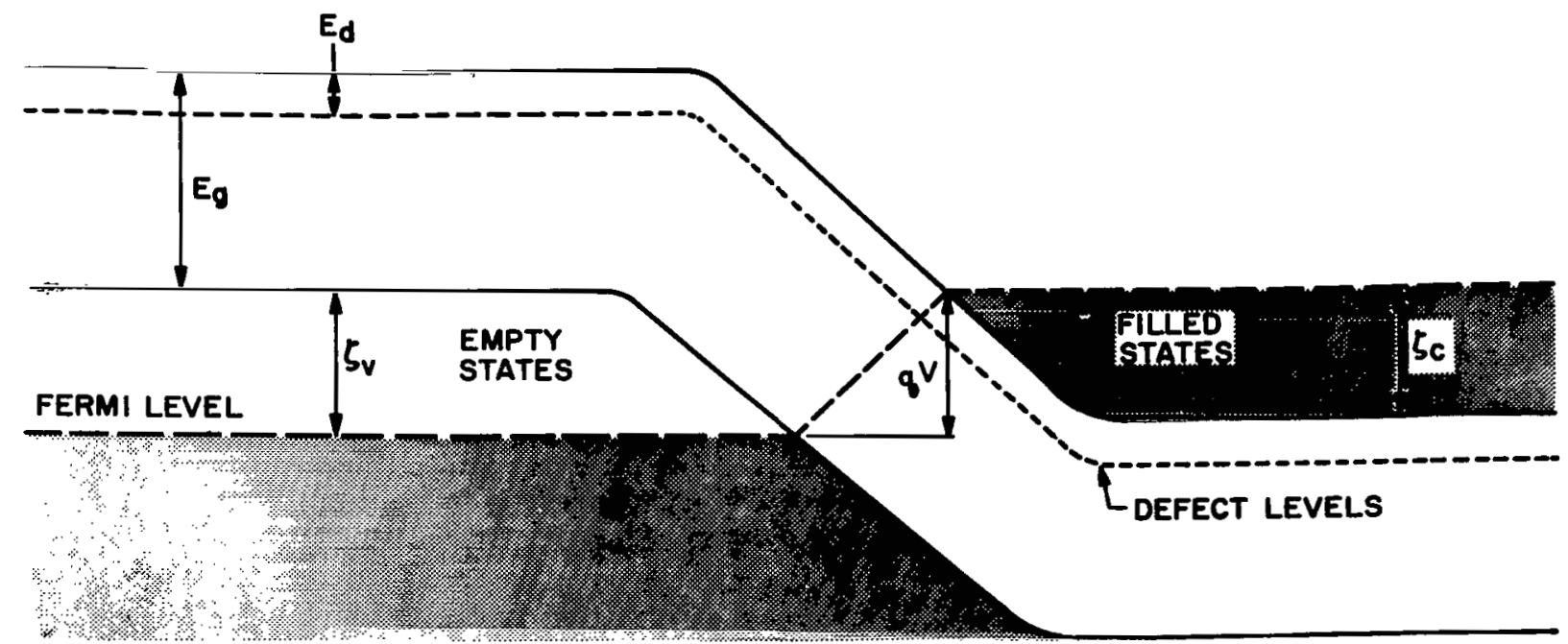

(a)

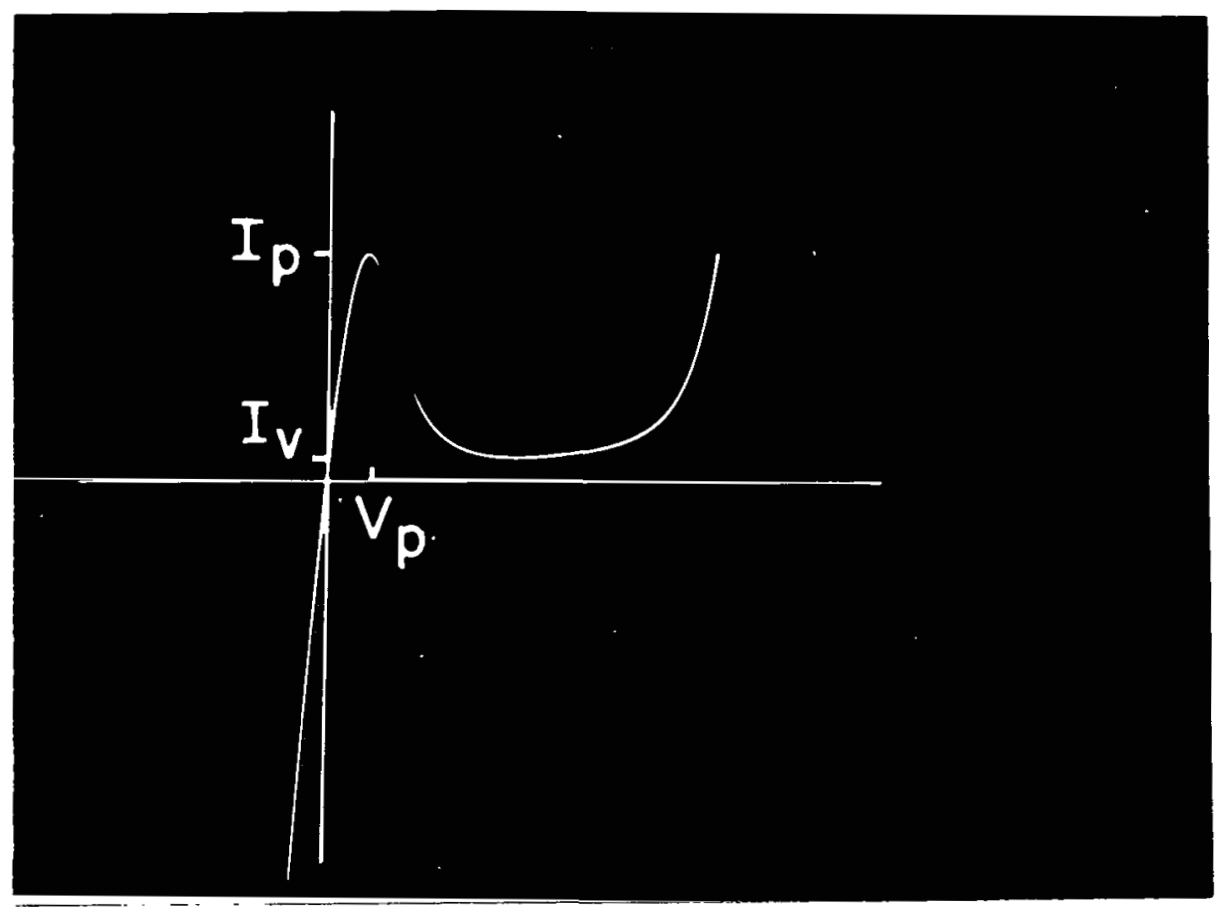

(b)

FIgure I. Energy band diagram (a) and I-V characteristic (b) of a tunnel diode. 


$$
I_{p} \sim \frac{\exp \left(-C^{\prime} E_{G}^{3 / 2}\right)}{E_{G}^{1 / 2}}
$$

For small hydrostatic pressure the band-gap varies linearly (ref. 11):

$$
\mathrm{E}_{\mathrm{G}}=\mathrm{E}_{\mathrm{GO}}+\mathrm{C}_{\mathrm{G}} \sigma
$$

where $\sigma$ represents pressure and $C^{\prime}$ and $C_{G}$ are material constants. Therefore, for small pressures

$$
\ln \frac{I_{p \sigma}}{I_{p}}=-3 / 2 c^{\prime} \quad C_{G} \sigma E_{G O}^{1 / 2}
$$

The magnitude of the hydrostatic pressure effect measured by Nathan and Paul (ref. 12) and the corresponding uniaxial pressure effects to be expected for various orientations are shown in Figure 2.

The valley current region is dominated by indirect tunneling via defect levels: electrons located at the most energetic filled defect state on the n-side tunnel to the valence band on the pside via a virtual state at the level $E_{d}$ below the p-side conduction band edge. Classen (ref. 13) has shown this excess current to be expressed by

$$
I_{x}=A D_{x} \exp \left[C\left(V_{F}-\frac{E_{G}+\zeta_{C}+\zeta_{v}}{e}\right)\left(\frac{E_{G}-E_{d}+\zeta_{v}}{E_{G}+\zeta_{C}+\zeta_{v}}\right)^{3 / 2}\right]
$$

where $D_{x}$ is the number of intermediate tunneling levels, $A$ is a constant which depends on junction area, and $C$ is a constant which depends on effective mass and junction width. The Fermi energies, $\zeta_{C}$ and $\zeta_{V}$, are defined in Figure 1 .

With the application of uniaxial stress the defect energy level changes linearly as does the bandgap (ref. 11).

$$
E_{d}=E_{d o}+C_{d} \sigma
$$

The value of $\mathrm{C}_{\mathrm{G}}$ is known for $\mathrm{GaAs}$ and $\mathrm{GaSb}$ (ref. II) ( $\mathrm{C}_{\mathrm{G}}$ for GaAs $=3.76 \times 10^{-6} \mathrm{eV} \mathrm{kg}^{-1} \mathrm{~cm}^{2}, \mathrm{C}_{\mathrm{G}}$ for $\mathrm{GaSb}=4.08 \times 10^{-6} \mathrm{eV} \mathrm{kg}^{-1}$ $\left.\mathrm{cm}^{2}\right)$. Studies of the pressure dependence of impurity levels in Ge, 


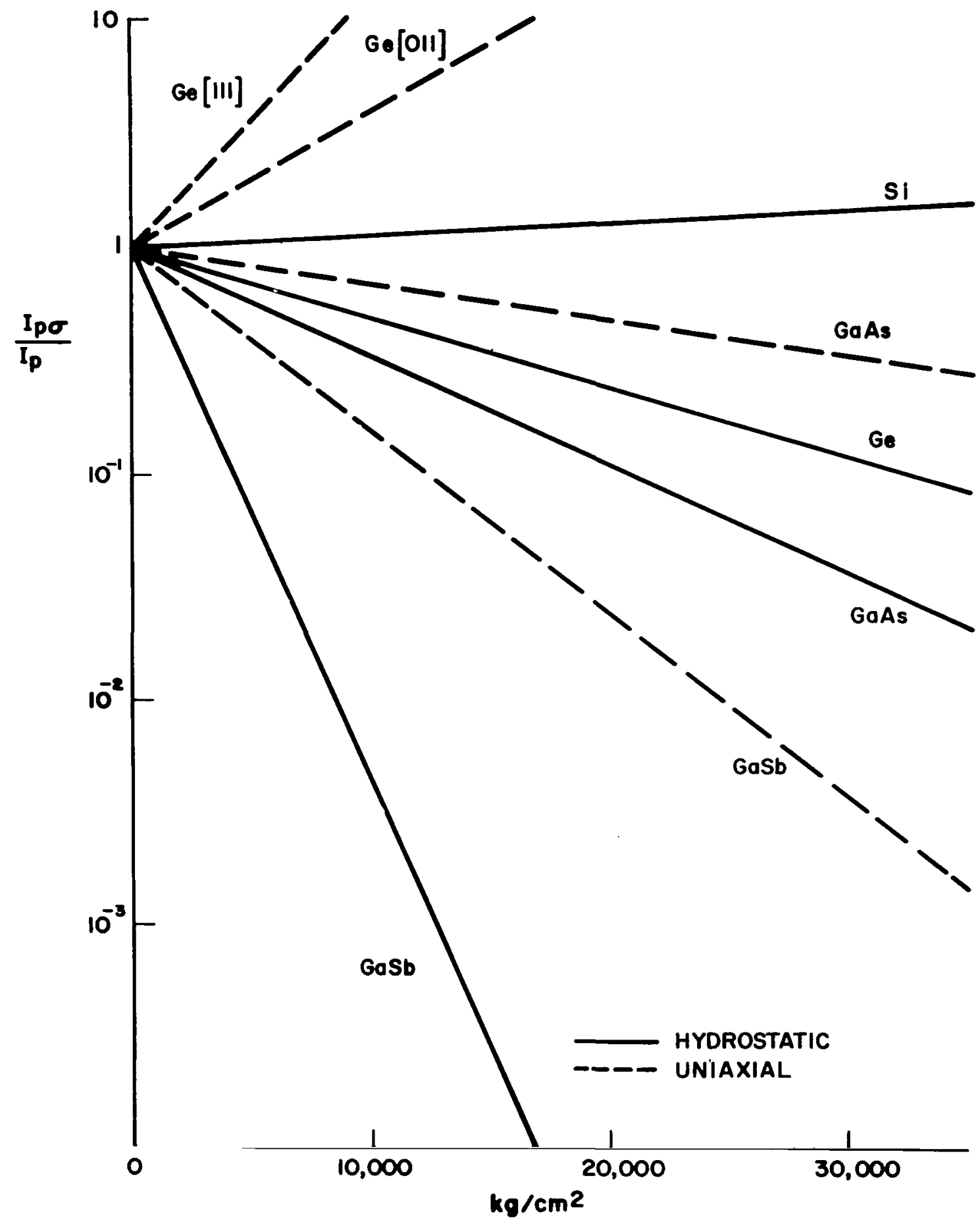

Figure 2.- Hydrostatic and uniaxial pressure dependence of the peak currents of Ge, Si, GaAs and GaSb tunnel diodes (after Nathan and Paul (ref. 12)). 
Si and GaAs (ref. 14) have led to the general conclusion that these levels have the same pressure dependence as the top of the valence band, i.e., $c_{d}=C_{G}$. Therefore, the bandgap mechanism would be expected to yield changes by about the same ratio for the valley as for the peak current (ref. 15). Figure 3 shows valley current changes we have observed, albeit not consistently, which are in reasonable agreement with the quantitative relationship expected from Eqs. (4) and (5).

Under high pressure and in the presence of mechanical damage, however, these relationships appear to be inapplicable (ref.llb). In these cases $I_{X}$ consistently and very substantially increases with pressure. Since $\mathrm{C}_{\mathrm{d}}=\mathrm{C}_{\mathrm{g}}>0$ in GaSb, there clearly is a conflict with the bandgap model both in magnitude and sign of the effer That these excess current effects are intimately associated with the presence of defects is clearly established by the results of the following experiment: a GaSb unit is subjected to a pressure of about $1300 \mathrm{~kg} / \mathrm{cm}^{2}$ (Figure $4 \mathrm{a}$ ). Both peak and excess current changes in roughly similar proportion and in the same direction as conventional theory would predict ("normal" effect). We now apply pressure beyond the elastic limit of the device, as evidenced by the small irreversible increase of excess current shown by the no-stress curve in Figure 4b. A pressure signal roughly equivalent to that applied before now yields a nearly 50 percent increase of excess current ("anomalous" effect). The sole, ad hoc, interpretation advanced so far for this effect is that stress modulates the number of active energy levels in the bandgap, relatively small variations in the number and position of effective levels being adequate to explain the magnitude of the effect (ref. II). Any practical applications of this large effect are severly limited by the associated large noise and instabilities which more than offset the advantages accruing from its magnitude.

Tunneling is basically a highly temperature-independent mechanism; however, even small temporature effects can be equivalent to significant pressure signals. While it might appear as if the temperature dependence could readily be deduced from Eq. (1), the implicit functional dependence of $E_{F}, E_{G}$, effective mass, etc., on temperature can completely override the explicit temperature dependence and hence, in practice, not even the sign of the temperature dependence can be predicted with any certainty. The only major controllable factor is the doping level, whose effect on temperature dependence has been analyzed in certain cases (refs. 16,17 ).

Since tunneling is an extremely fast process, any frequency limitation encountered in the TDT can only be of mechanical origin. Here again, the small and intricate structure of the TDT makes any theoretical analysis a purely academic undertaking and thus 


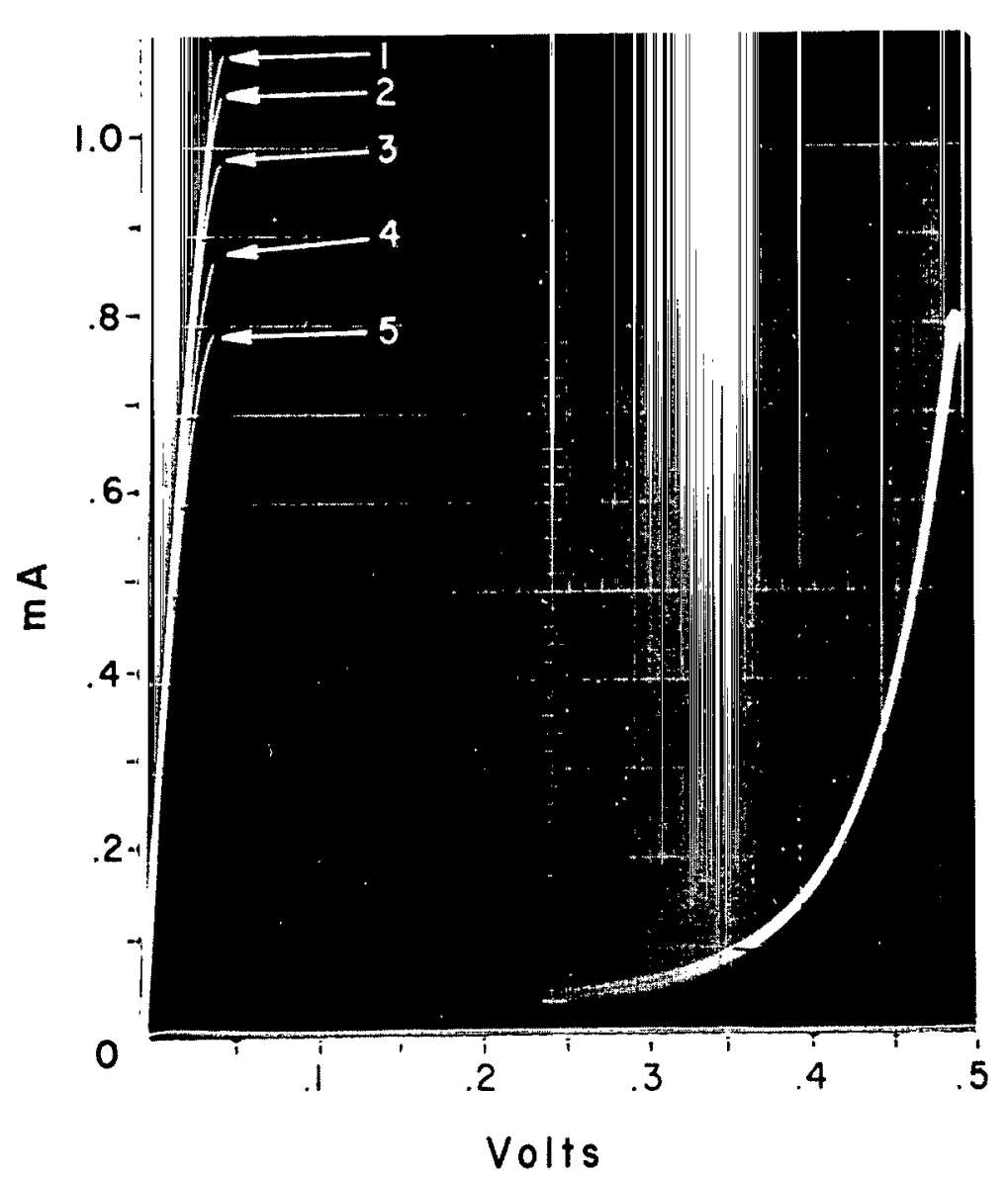

(a)

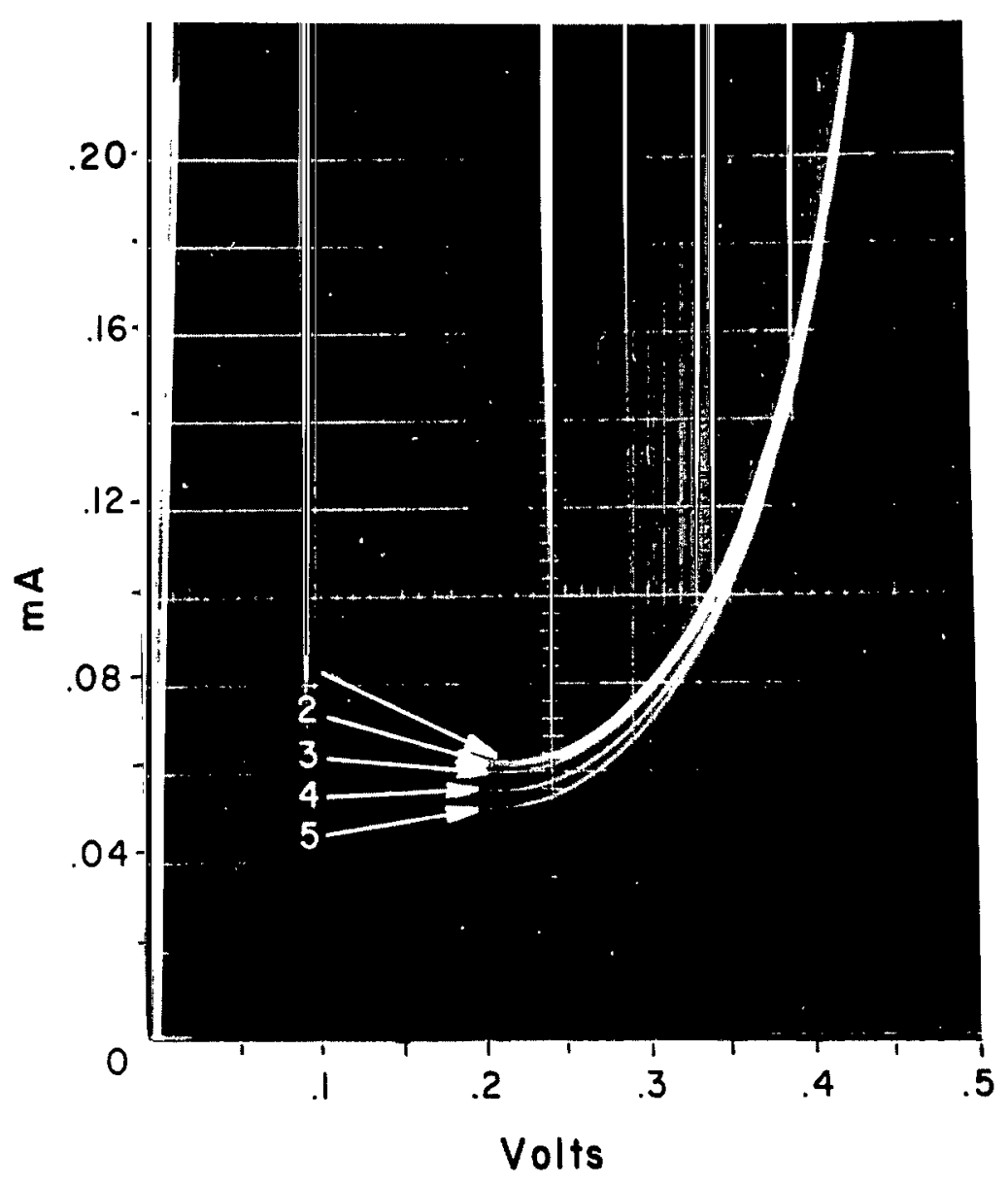

(b)

Figure 3.- Peak (a) and valley current (b) changes under uniaxial compression. The numbers correspond, in order, to 0 , $200,600,1300$ and $1800 \mathrm{~kg} / \mathrm{cm}^{2}$, approximately. 


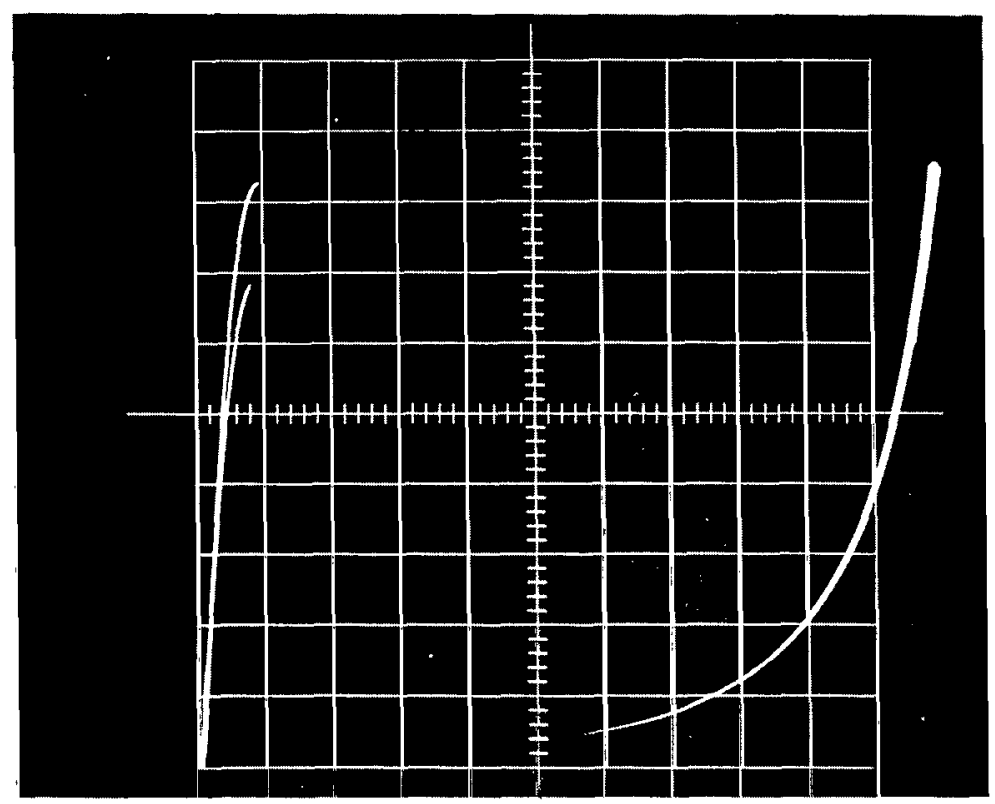

(a)

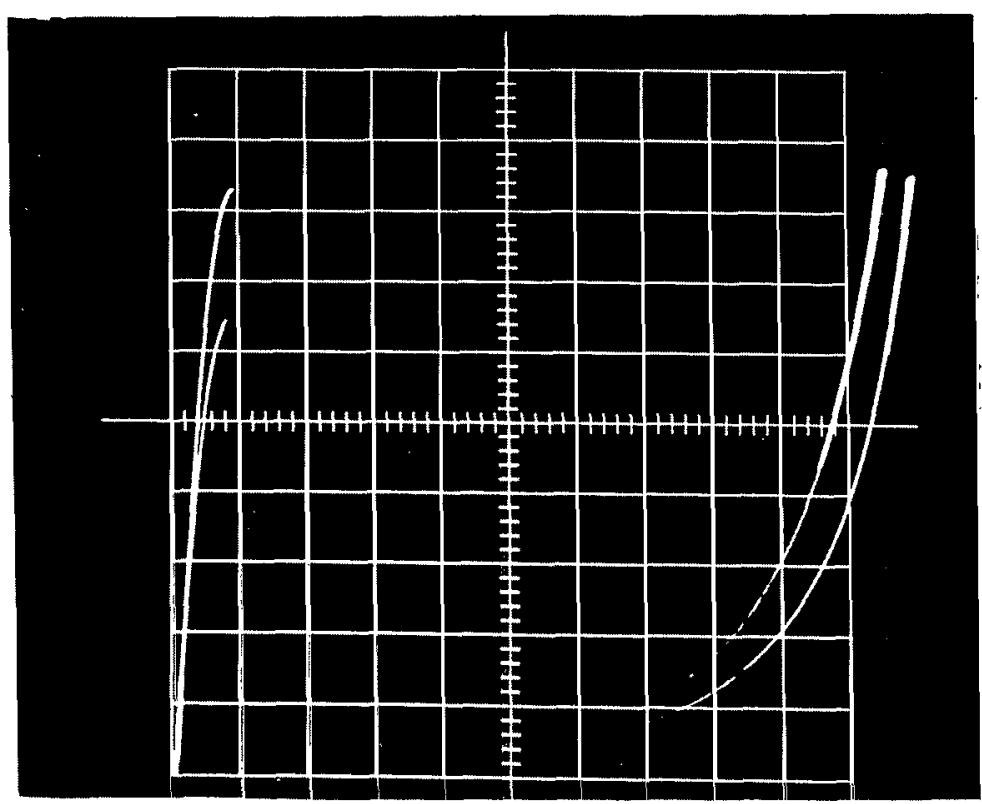

(b)

Figure 4.-

Vertical: $\quad 100 \mu \mathrm{A} / \mathrm{div}$

Horizontal: $50 \mathrm{mV} / \mathrm{div}$

(a) "Normal" effect of uniaxial pressure on a GaSb tunnel diode. (b) "Anomalous" effect of about the same pressure on the diode, following mechanical overstressing. Note the large reversible excess current increase while the peak current shows the normal decrease. 
experiment rather than theory must, for the time being, provide design guidelines.

\section{DEVICE FABRICATION}

The TDT concept lends itself to realization in a variety of electromechanical devices. Our own efforts were specifically aimed at the development of miniature pressure transducers, although we have also given attention to force transducers and accelerometers, using one basic design with only relatively minor variations. This design was based on existing commercial tunnel diodes ( $\mathrm{Zn}$-doped p-GaSb with a Te alloy) with the primary objective of establishing practicality of the device concept; engineering refinement was given relatively little attention. Gasb was chosen as the device material because we found it to yield good pressure sensitivity and tolerance to manipulation during fabrication.

The device housing is a conventional (but uncapped) microwave pill package with two cuts parallel to the device axis (Figure 5). The cuts are made to reduce device dimensions and to provide a flat surface for deposition of a stabilization resistor.

Generally, we found it desirable to reduce the mesa crosssection by standard electrolytic etching. For the particular diodes used, a peak current of 0.5 to $1 \mathrm{~mA}$ was chosen as a measure of adequately small junction diameter. Figure 6 shows the effect of etching on the mesa configuration. The mushroom-shaped structure, considerably larger in diameter at the top than at the neck where the junction is located, makes it clear that the high stresses are concentrated there.

Following etching, a shunt resistor is applied to one of the two flat sides of the package (Figure 5). The resistor is a vacuum-deposited thin $(\sim 200 \AA)$ Cr layer, about $1 \mathrm{~mm}$ wide and extending over the two electrodes of the device. The resistance (50 to 100 ohms), corresponding to the negative slope of the I-V characteristic, is monitored during evaporation, with due allowance for the approximately 10 percent increase following exposure to the atmosphere. The film is then covered with a thin protective coating of hard epoxy.

The mechanical coupling between the top of the mesa and the sensing surface (a gold-plated beryllium-copper disk) is accomplished by means of an epoxy column (Figure 7). The disk, which has a center hole, is epoxy-bonded to the periphery of the structure and, through the hole, to the coupling column, thereby allowing operation both in tension and compression without preload. It should be noted that the stresses in the epcxy bonas are very moderate compared with those at the junction. 


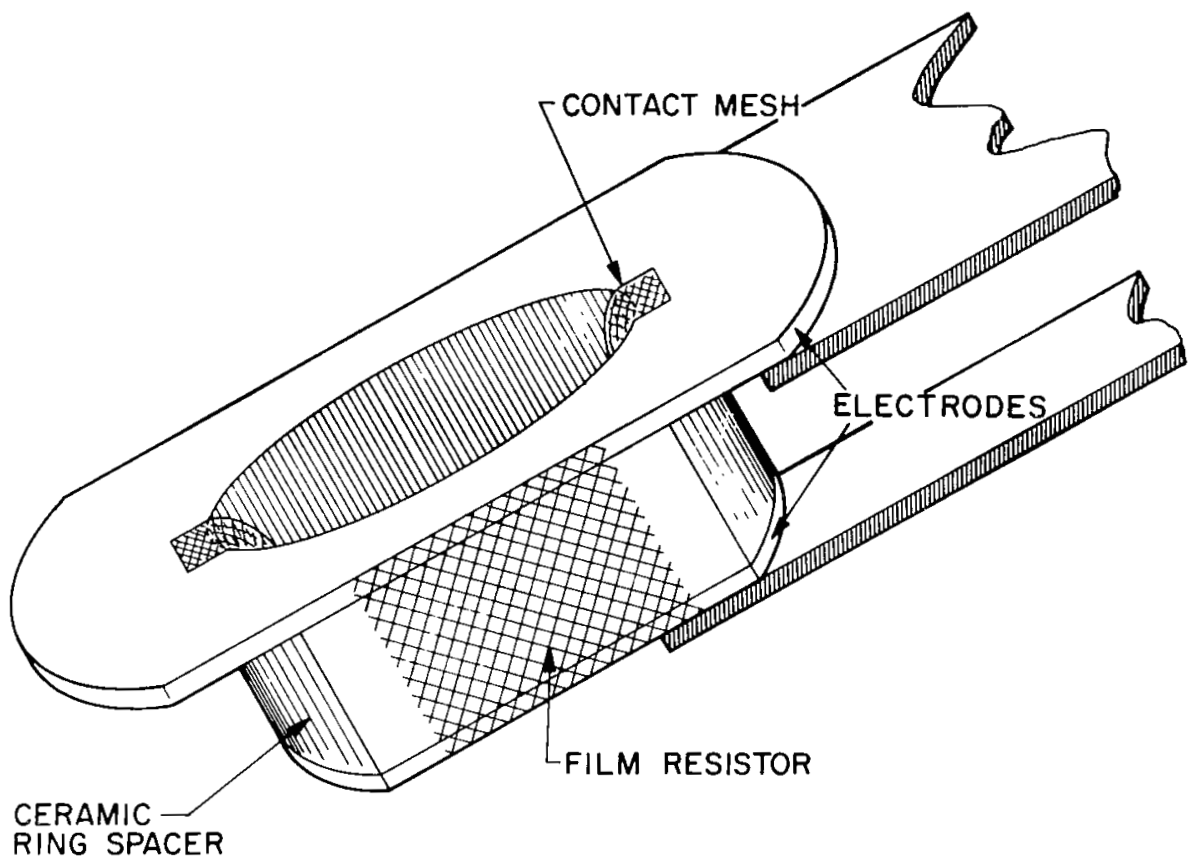

Figure 5.- Housing and electrode configuration of a TDT. Note the evaporated Cr-film stabilizing resistor.
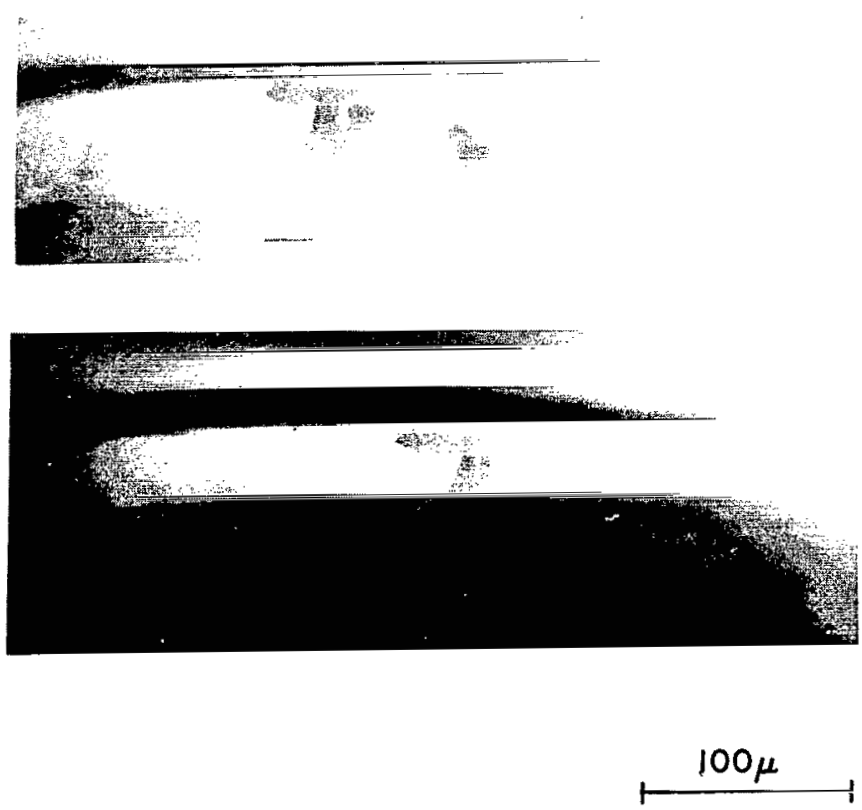

Figure 6.- Microphotographs of a tunnel diode mesa (and the top electrode) at two successive etching stages. The typical TDT mesa size is indicated in the lower photograph. 
For application as a cardiovascular pressure transducer, a small ridge of epoxy is built up around the device to provide a measure of protection for the sensing diaphragm. A thin coaxial cable ( $13 \mathrm{mils}$ ) is attached to the transducer; a stainless-steel sleeve is slipped over the device, and the assembly is bonded to the tip of a catheter (Figure 8). In an alternative construction, also shown in this figure, an epoxy coating is substituted for the stairless-steel sleeve. The insert depicts a TDT whose critical diameter is about $0.5 \mathrm{~mm}$. For such small devices the ceramic spacer is actually cut open (Figure 7) and the housing is subsequently sealed by means of epoxy-bonded sidewalls. The film resistor is deposited on one of the exposed flat ceramic surfaces. To take full advantage of the minute size of the device, thin polyethylene tubing $(0.6 \mathrm{~mm}$ OD) had to be used as a catheter.

We have used a basically similar construction for experimental accelerometers which incorporated a spherical seismic mass of 13 milligrams bonded directly to the mesa. No attempt was made to reduce cross axis modulation by means of lateral support, since the purpose of these accelerometers was only to provide a convenient means to study frequency response.

\section{DEVICE CHARACTERIZATION}

Device evaluation was conducted on "normal" units only: "anomalous" units (that is, those showing large pressure dependence of the excess current - several times larger than that of the peak current) were rejected. This selection was based on extensive experience which proved such devices to be noisy and erratic. As pointed out, it appears that these large "anomalous" pressure effects, which in GaSb are of opposite sign to that of the "normal" effect, stem from a mechanism associated with crystal defects.

The "normal" excess current effect, i.e., the effect involving a relative current change of about the same magnitude as that of the peak current, and of the same sign, does offer certain operational advantages, as outlined in the Discussion. * However, we have not investigated these to any significant extent and we have concentrated, instead, on the normal peak current effect.

To avoid instabilities inherent in operation at the peak voltage, stabilization by means of a shunting resistor in close proximity to the junction (for minimum series inductance) is required. We have satisfied this condition by vapor deposition of a thin film resistor directly onto the tunnel diode housing, as described in the section on device fabrication.*

The tests were performed with the TDT connected to a conventional dc differential amplifier. For static tests we used a small pressure chamber monitored by a reference transducer (MKS Baratron type 77) whose output, together with that of the TDT, was fed to an $\mathrm{X}-\mathrm{Y}$ recorder.

\footnotetext{
* Page 22

* Page 9
} 


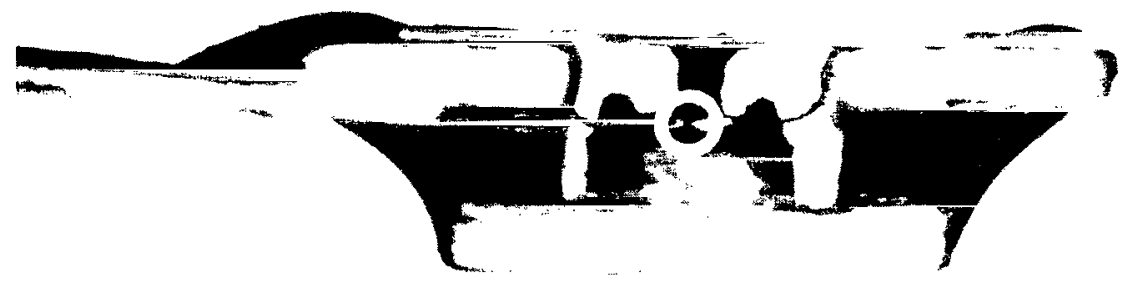

Figure 7.- Cross section of a TDT, complete except for sidewalls. The circled area shows the critical interface between mesa and epoxy column.

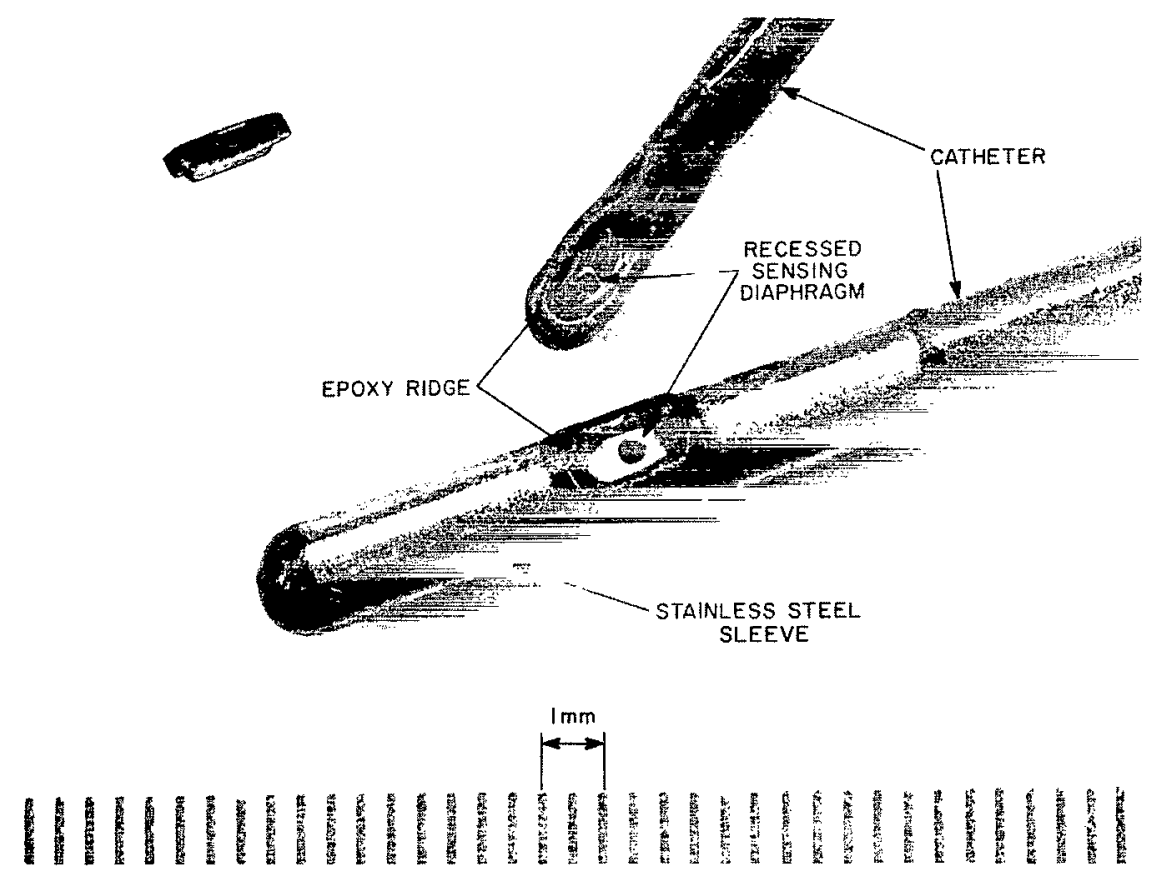

Figure 8.- Two catheter-mounted TDT's designed as experimental cardiovascular pressure transducers. Insert shows a $0.5 \mathrm{~mm}$ wide TDT. 
Figure 9 shows a typical result of such a measurement extending over both positive and negative pressures. (The sensitivity is about $12 \mu \mathrm{V} / \mathrm{mm} \mathrm{Hg}$ and the resolution about $0.5 \mathrm{~mm} \mathrm{Hg}$. Linearity over the $300 \mathrm{~mm} \mathrm{Hg}$ range is within +6 percent).

To simplify the discussion of the results, we shall make use of an expression relating the signal voltage $\Delta \mathrm{V}$ across the TDT to the pressure signal $\Delta \sigma$

$$
\Delta V=\left(\frac{\delta I}{\delta \sigma}\right)\left(\frac{\delta V}{\delta I}\right)_{\substack{I=I_{O} \\ \sigma=\sigma_{0}}} \Delta \sigma
$$

where the first coefficient represents the pressure dependence of the current and the second coefficient $\delta \mathrm{V} / \delta I=r_{d}$ is the differential resistance (comprising device, shunt, and load resistance). Clearly this expression is valid only for small-signal conditions, but it represents an adequate appproximation for our present purposes.

From Figure 10 which shows the stabilized I-V characteristic in the range of interest $1 / 2 \mathrm{~V}_{\mathrm{p}}$ to $2 \mathrm{~V}_{\mathrm{p}}$, it is evident that $r_{\mathrm{d}}$ in Eq. (6) represents the dominant factor since it changes by well over an order of magnitude, compared to a $\delta I / \delta \sigma$ change by a factor of about 2 only. Clearly then, bias represents the most important parameter for the operation of the device. For example, a bias change of only $20 \mathrm{mV}$ can cause the sensitivity to vary by a factor of 4 (Figure 11).

Eq. (6) tacitly assumes $r_{\mathrm{d}}$ to be constant; however, since $r_{\mathrm{d}}$ is in fact pressure-dependent, non-linearities have to be expected. Ordinarily the non-linearities would range from 5 to 10 percent (Figure 11). In extreme cases (TDT biased in the negative conductance region) higher values have been observed.

Since the noise level is proportional to the differential resistance, as is the signal voltage, it is clear that the signal-to-noise ratio should be largely independent of bias over the normal operational range of the TDT. Our observations confirm this and also prove that the noise stems from the diode itself rather than from the associated circuitry. 


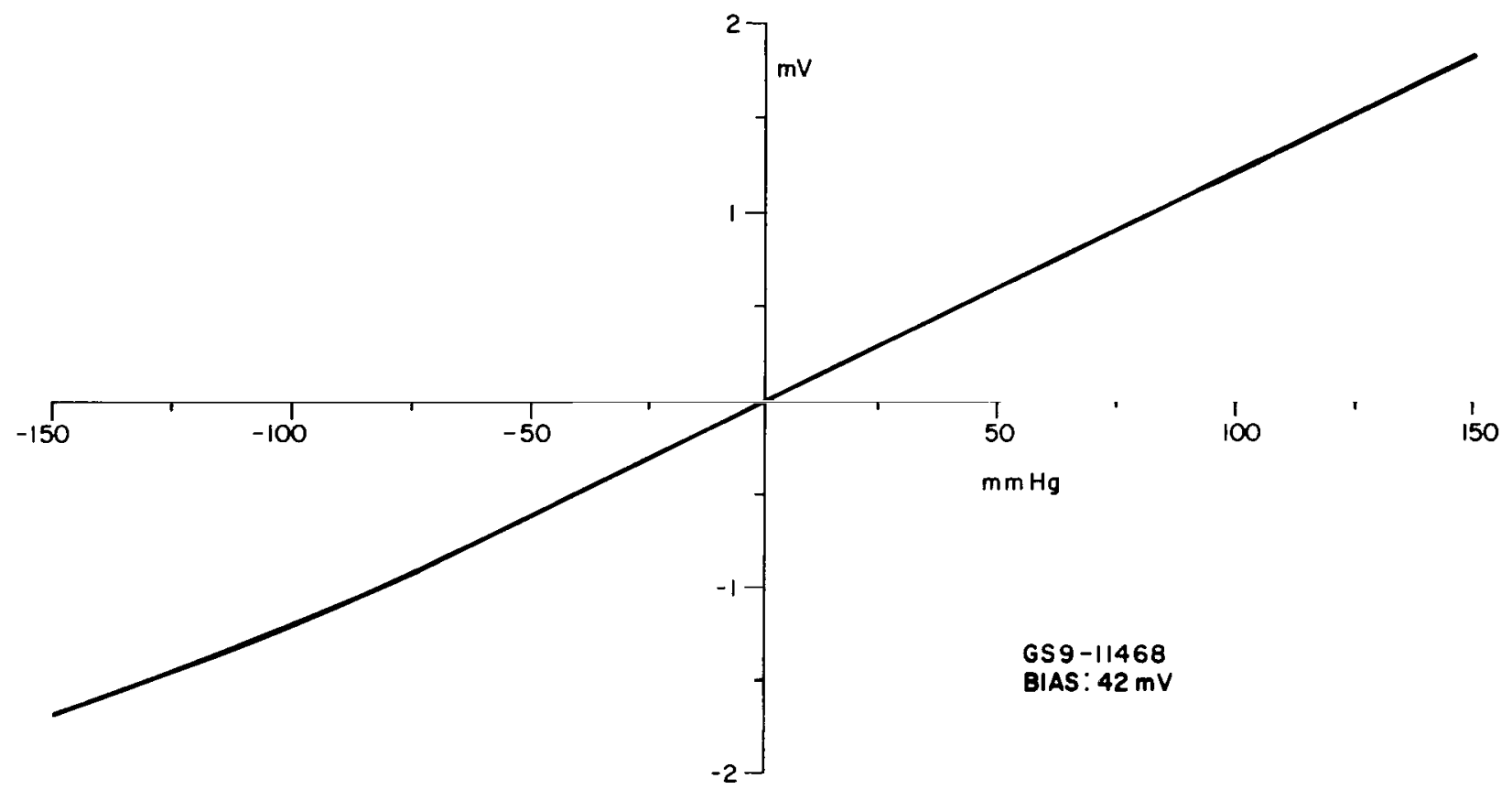

Figure 9.- TDT output voltage as a function of pressure.

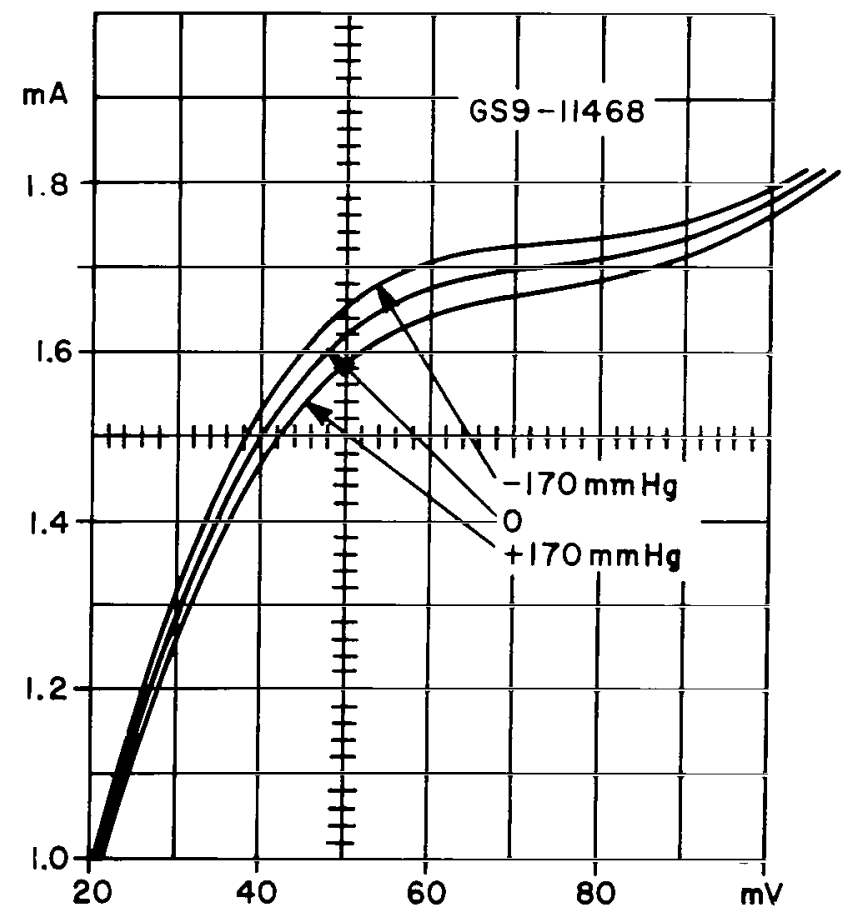

Figure 10.- Effect of positive and negative pressure on a stabilized TDT I-V characteristic. 
There is a close analogy between pressure and temperature effects in the TDT, as shown in Figure 12. A temperature change will again cause a bias shift with all the attendant effects discussed before. Figure 13 shows output as a function of pressure at constant-current bias, with temperature as a parameter. For ease of comparison all curves are offset along the ordinate in order to pass through the origin, with the actual zero-pressure bias indicated. The following features are readily apparent: the dominant effect is the shift of bias voltage (in this case equivalent to about $25 \mathrm{~mm} \mathrm{Hg} /{ }^{\circ} \mathrm{C}$ ), the pressure sensitivity over the $20^{\circ} \mathrm{C}$ interval varies quite considerably (by a factor of about 4 ) and goes through a maximum (at $34^{\circ} \mathrm{C}$ ), and the shape of the curve varies continuously from concave to convex. The explanation for this behavior follows readily from two basic relations, the dependence of bias on temperature (Figure 13) and the dependence of the differential resistance on bias (Figure 10). As temperature increases the bias increases, which in turn causes the differential resistance first to increase and then to decrease. As evident from Eq. 6, this variation of differential resistance gives rise to corresponding changes in sensitivity. (The temperature at which the sensitivity goes through a maximum can be chosen arbitrarily by appropriate selection of the shunting resistance.) Finally, the concave and convex shapes are consequences of the pressure dependence of the differential resistance and the resultant changes in sensitivity. At constant-bias voltage $V_{p}$, on the other hand, it is indeed found that the differential resistance, and hence the sensitivity, remain largely independent of temperature, as shown in Figure 14. Thus a sensitivity variation of about 10 percent results in this case, compared with a change of 400 percent in the constant-current case illustrated in Figure 13 .

The noise level of the TDT was established by direct observation on an oscilloscope. Typically, the peak-to-peak noise ranged between equivalent pressures of 0.3 to $5 \mathrm{~mm} \mathrm{Hg}$ for a $1000 \mathrm{~Hz}$ bandwidth. The noise spectrum, measured with a wave analyzer (GR Model 1900-A), showed essentially $1 / f$ dependence up to tens of $\mathrm{kHz}$ in agreement with previous results (ref. 18). The dynamic range of various TDT's with upper pressure limits between several hundred $\mathrm{mm} \mathrm{Hg}$ and several atmospheres was typically 50 to $60 \mathrm{~dB}$.

A special pressure chamber (ref. 19) was used to establish the frequency dependence of the TDT. We found that the frequency response was flat over the range of the instrument, from dc to $2 \mathrm{kHz}$. Flat frequency response was also measured up to about $4 \mathrm{kHz}$, using an accelerometer embodiment of the TDT.

Short-term stability is illustrated in Figure 15 which shows a strip-chart recording of the TDT output for a pressure signal applied over a period of about 10 minutes. Rise and fall times shown do not reflect device limitations, but the time constants of the pressure system. Tests conducted by periodic application 


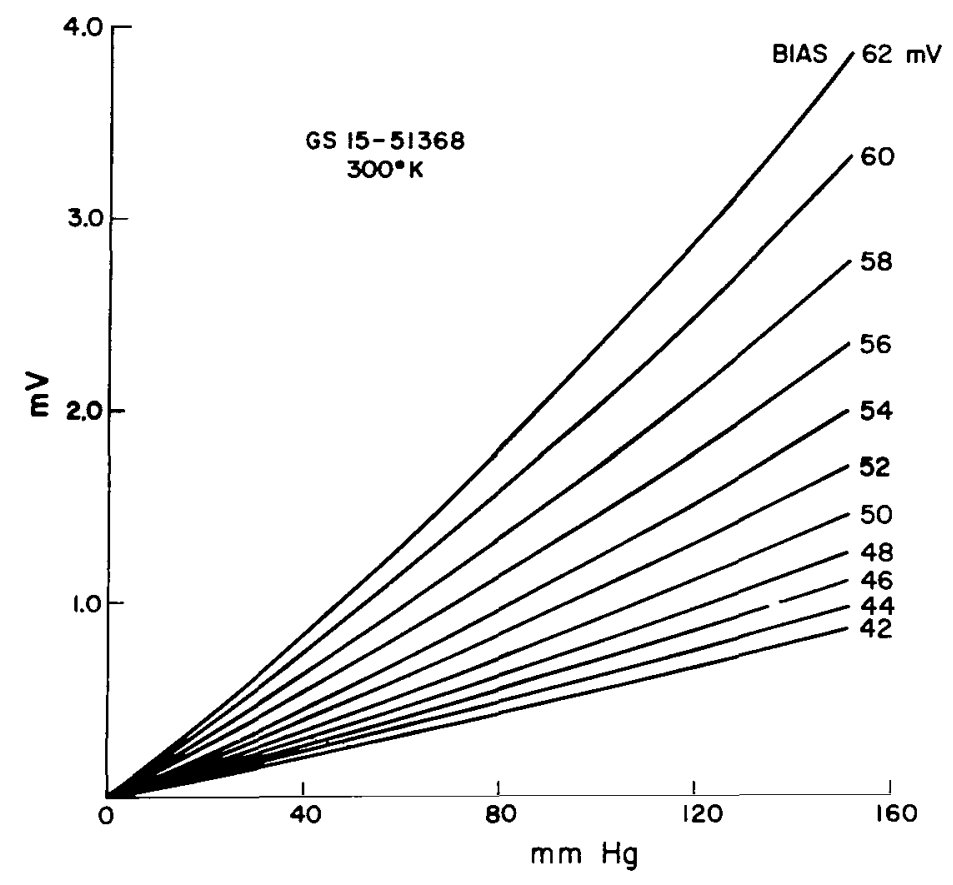

Figure 11.- TDT output voltage as a function of pressure with bias voltage as a parameter.

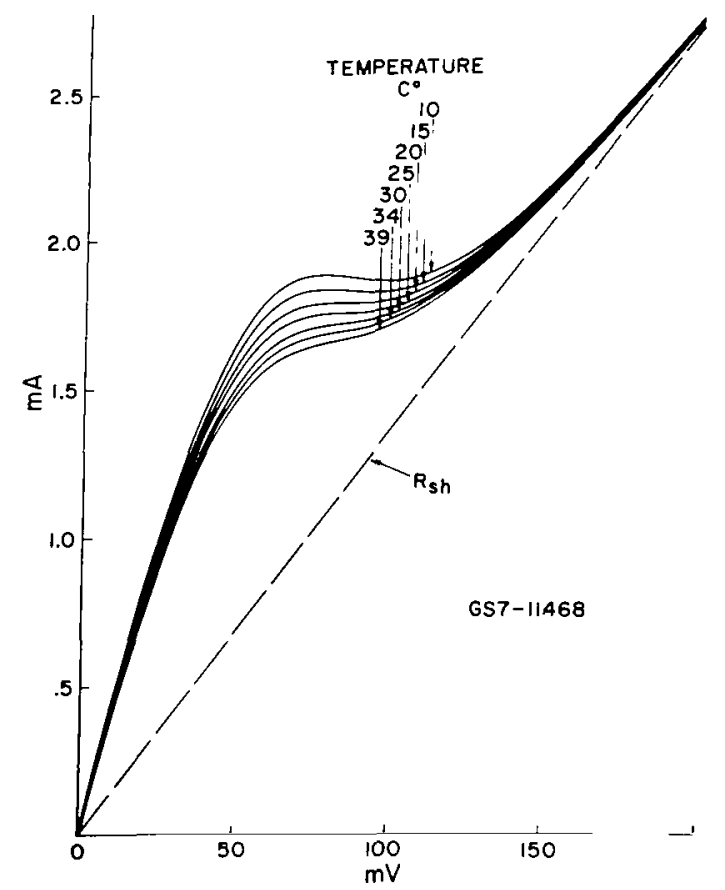

Figure 12.- Effect of temperature on a stabilized TDT I-V characteristic. The dashed line represents the stabilizing shunt resistor. 


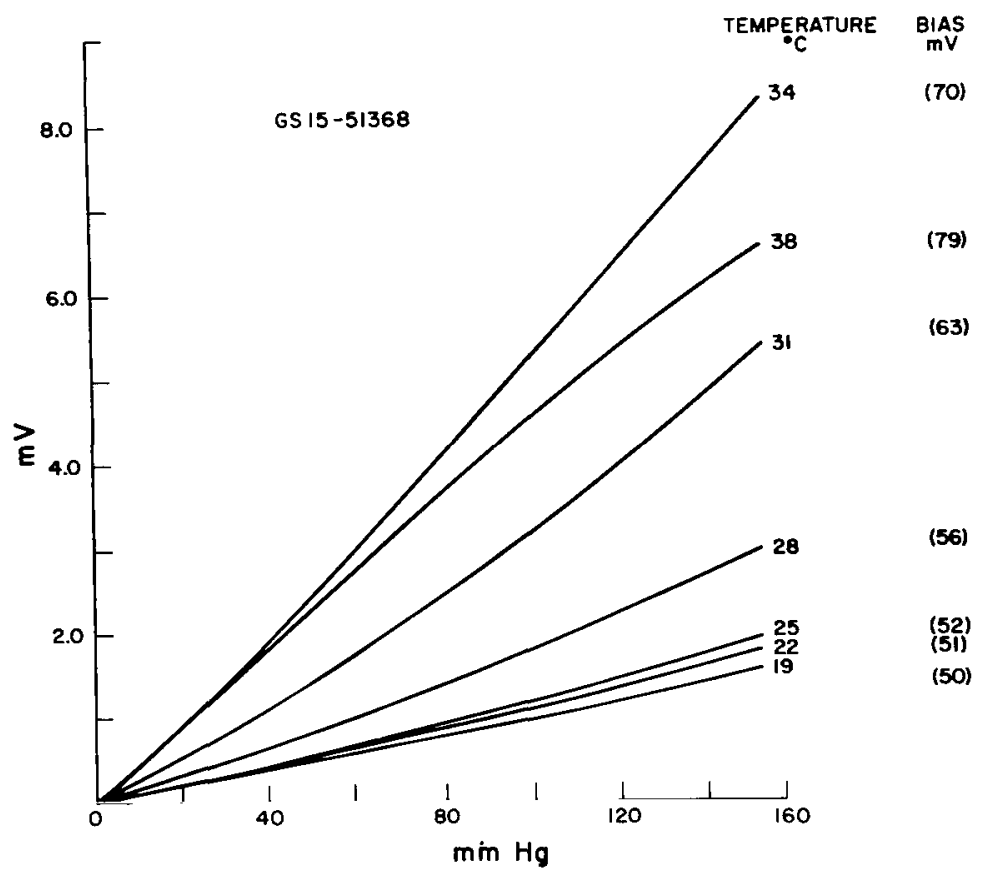

Figure 13.- TDT output voltage as a function of pressure with temperature as parameter. The zero-pressure bias voltages are shown in parentheses.

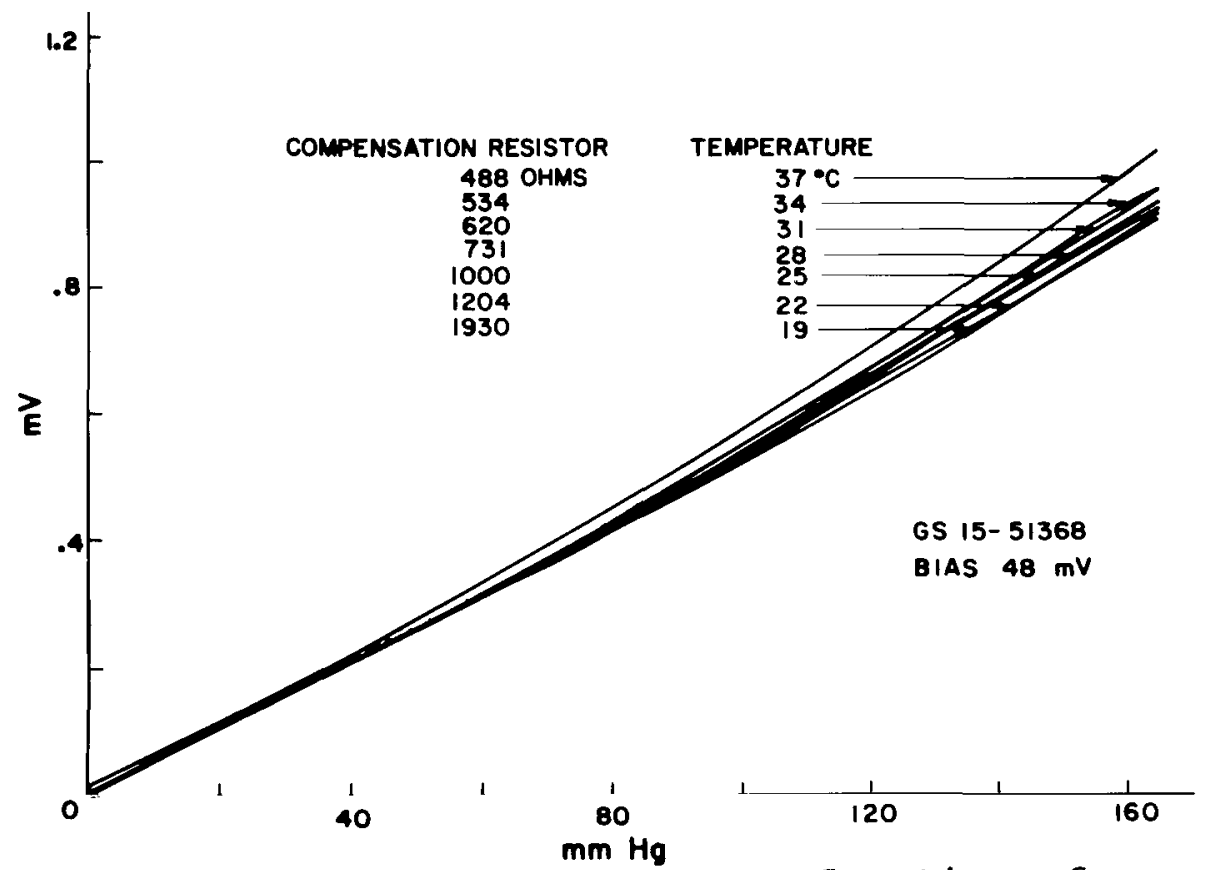

Figure 14.- TDT output voltage as a function of pressure with temperature as parameter. (The bias voltage is kept constant by means of a shunting compensation resistor.) 
of fixed pressure signals over periods of months showed reproducibility of output within +5 percent.

As an accelerometer with a 13 milligram seismic mass and a total mass of 60 milligrams, the device performed as shown in Figure 16. The resolution is about $0.5 \mathrm{~g}$, the sensitivity about $.14 \mu$ amps $/ g$ with a linearity of +8 percent. However, because of the poor lateral constraints of the seismic mass, cross axis sensitivity was about 40 percent. (The latter shortcoming should be readily correctable by appropriate mass and constraint design.)

The next two figures illustrate some of the TDT performance characteristics observed in practical tests conducted by B. Lown and $R$. Cannon of the Harvard School of Public Health. Figure 17 shows operational results of the device in an experiment in which pressure and its first and second derivatives were to be measured in the left ventricle of the heart of an animal. In Figure 18 the fidelity of the TDT is compared with that of one of the most advanced experimental cardiac pressure transducers, the Fiberoptic catheter device (ref. 20). The two outputs, shown displaced with respect to each other, display no significant difference in their fine structure.

\section{TDT CIRCUITRY}

The negative resistance characteristic of the TDT permits operation in stabilized, oscillatory or switching modes. A number of different circuits for one or the other mode have been described in the literature (refs. 9, 21). Of these we found particularly useful the stabilized mode in which the TDT acts essentially as a pressure controlled (non-linear) resistor across which the signal voltage is developed. Temperature compensation in this mode is readily achieved by standard techniques, as illustrated in Figure 19: using a balancing tunnel diode in conjunction with a differential amplifier, the temperature dependence of the TDT is reduced from the equivalent of $50 \mathrm{~mm} \mathrm{Hg} /{ }^{\circ} \mathrm{C}$ (dashed curve) to about $\pm 1 \mathrm{~mm} \mathrm{Hg} /{ }^{\circ} \mathrm{C}$.

For the switching mode we have developed a simple circuit which directly utilizes the negative resistance of the TDT. In this circuit pressure-induced changes in the peak current provide pulse-width modulation. The TDT is driven by a current source whose output consists of a sawtooth wave superimposed on a dc component and a reset pulse. This current drive gives rise to a voltage pulse whose duration (and shape) is a function of pressure. Figure 20 illustrates this mode of operation and should require no further elaboration. The same scheme can readily be adapted to provide conversion of pressure input into frequency variation, and hence into digital output, by means of the arrangement in Figure 21. The TDT voltage pulse is differentiated and the resulting pulse, 


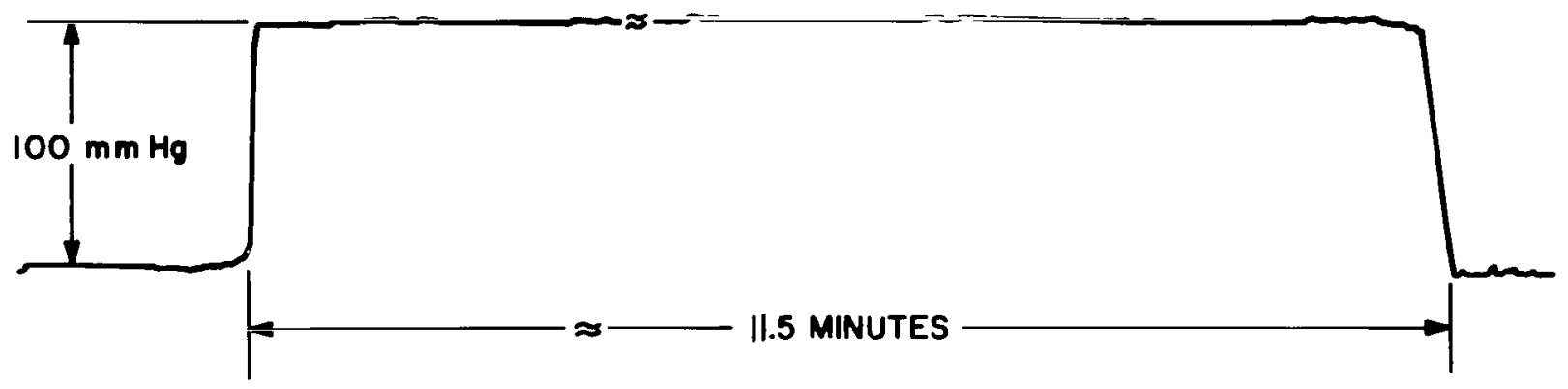

Figure 15.- Short-term stability of a TDT. Rise and fall times are those of the signal input.

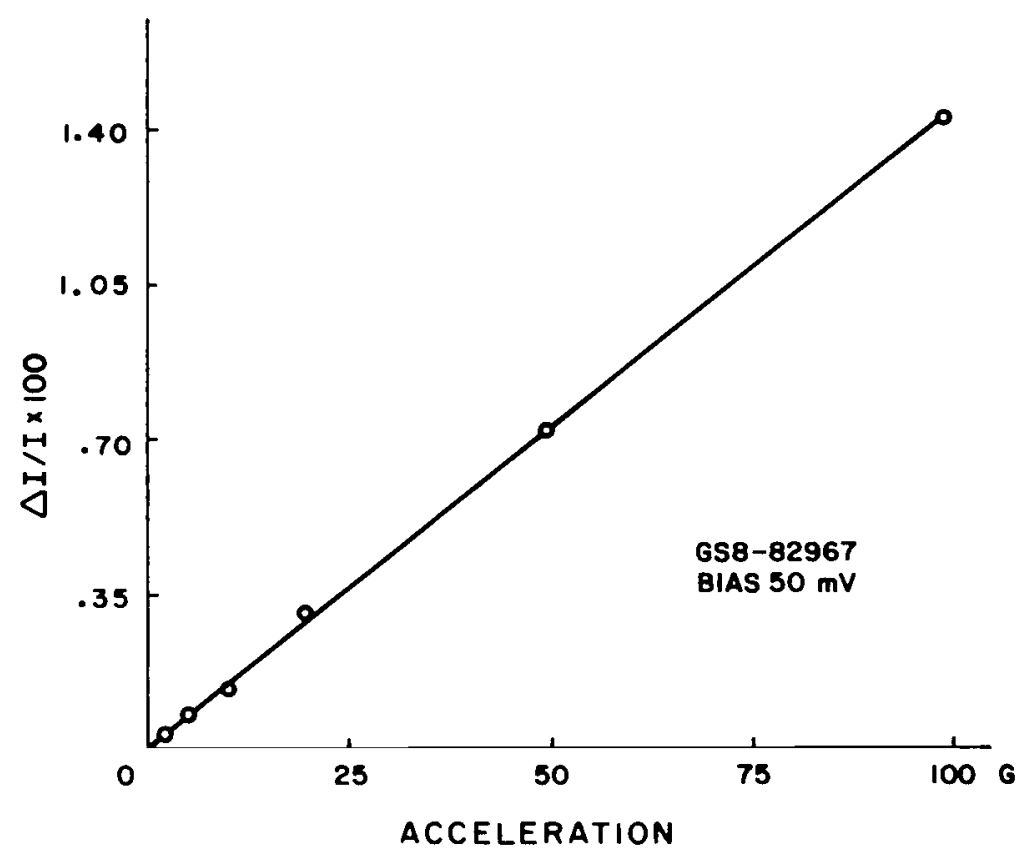

Figure 16.- TDT accelerometer output $(1000 \mathrm{~Hz})$. 


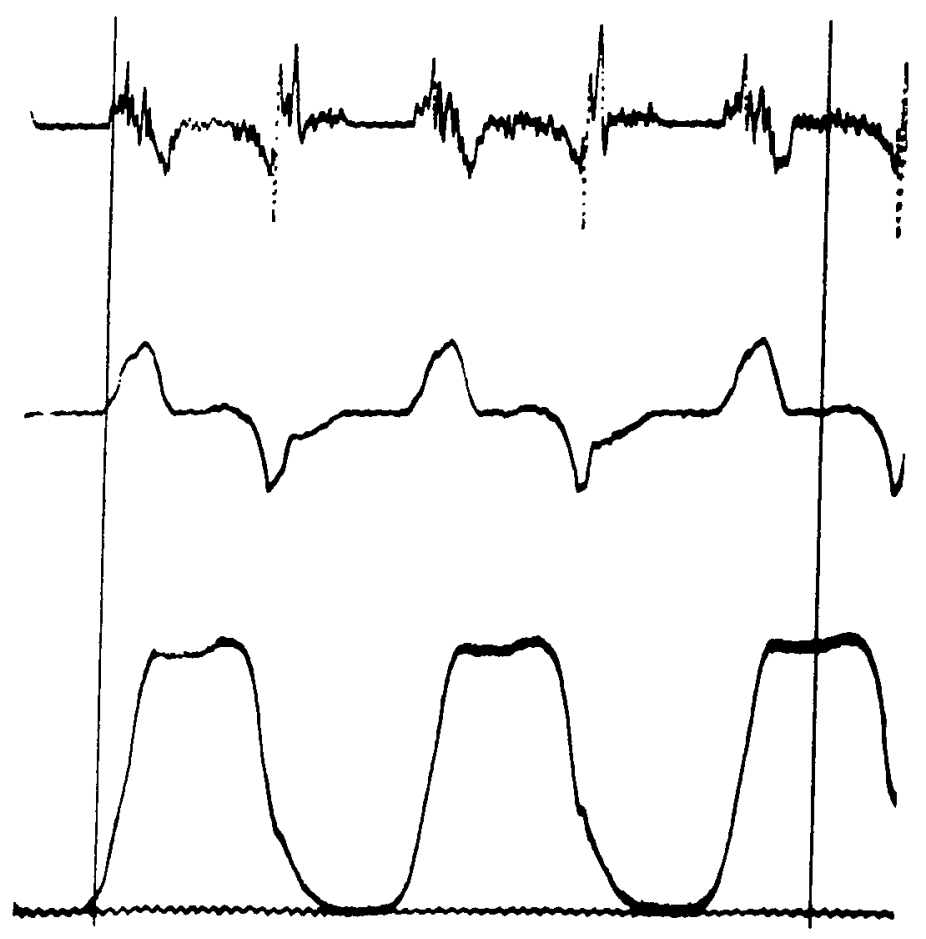

Figure 17.- Left ventricular pressure measured with a TDT. From bottom to top the curves represent pressure and its first and second derivations.

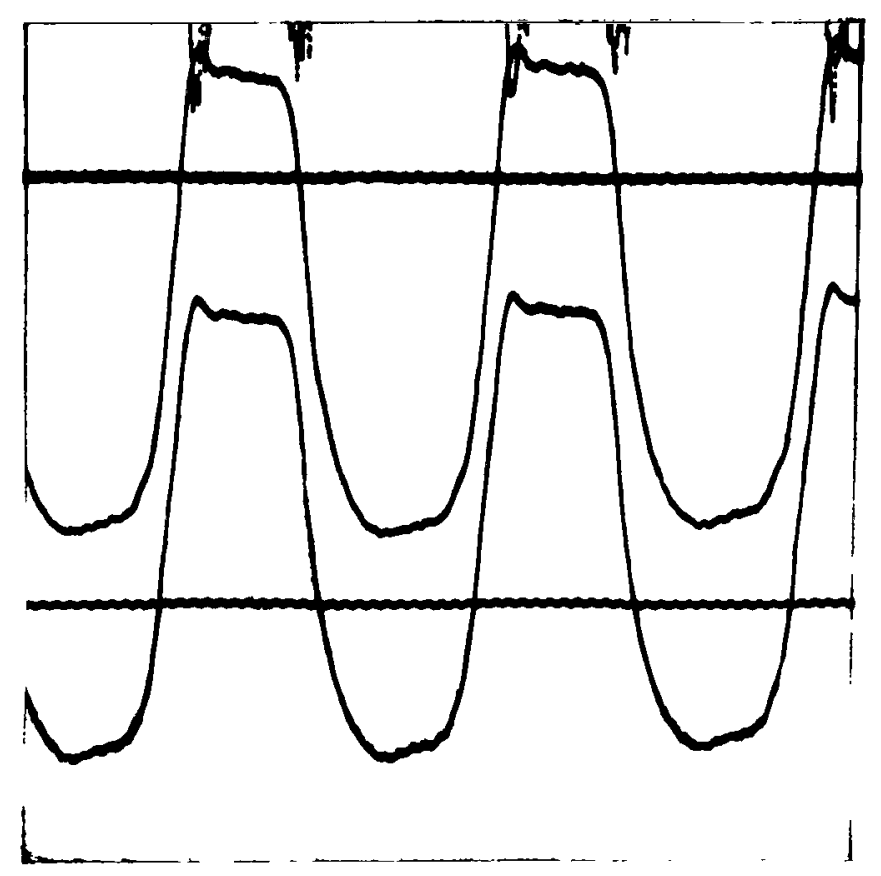

Figure 18.- Ventricular pressure signals obtained with a TDT (bottom) and a Fiberoptic transducer. 


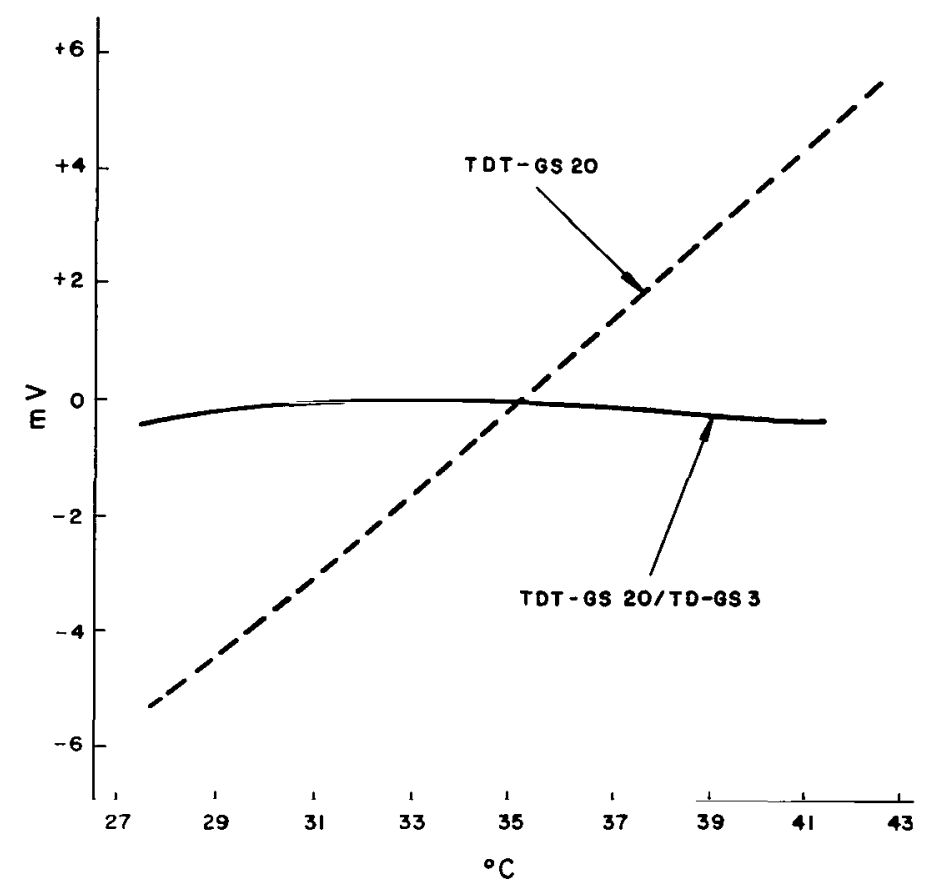

Figure 19.- Compensated (solid curve) and uncompensated bias voltage shift with temperature.

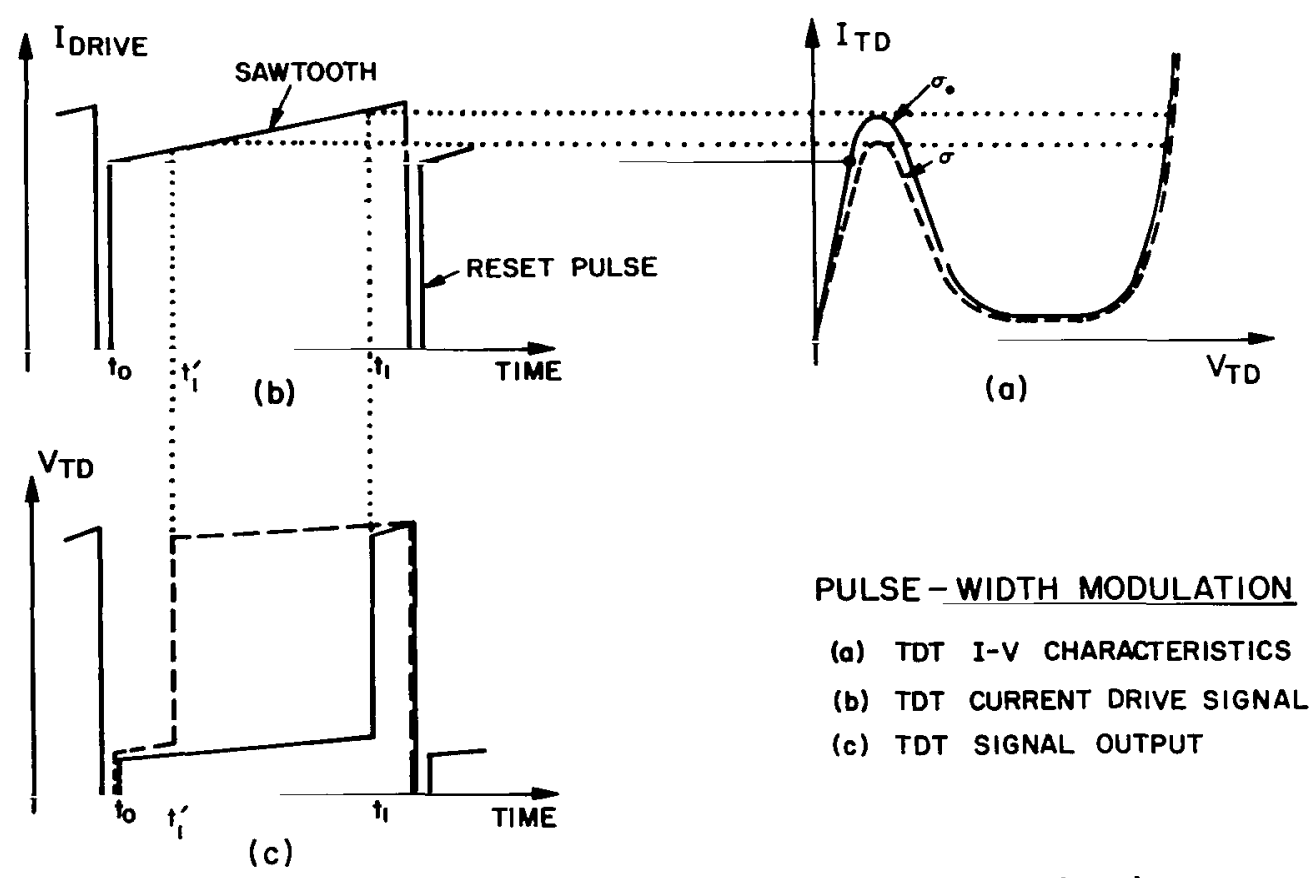
Figure 20.- Schematic pulse width modulation
scheme for the TDT. 
generated by switching from the low to the high voltage state, is used as a trigger for the current-drive pulse generator.

A circuit implementation of the PWM and FM modes, and of a small transmitter, is shown in Figure 22. The pulse generator is a complementary astable multivibrator. With transistors $Q_{1}$ and $Q_{2}$ in the off-state, the discharge current from $C_{1}$ gives rise to a quasi-linear ramp voltage across $\mathrm{R}_{1}$. This voltage is coupled to the current drive stage $Q_{3}$, the collector of which drives the TDT. With the mode switch in the FM position the differentiated TDT output, fed back to the multivibrator, provides the required trigger signal. The resulting FM signal, taken from the collector of Q1, is used to modulate the transmitter. The circuit is designed for a minimum power drain from a single mercury cell supply. Figures 23 and 24 show the PWM and FM signal outputs of this circuit. The trace at the top of Figure 23 represents zero pressure and the bottom trace a PWM signal of $150 \mathrm{~mm} \mathrm{Hg}$. It is worth noting that the 100 percent PWM modulation was obtained with less than 2 percent change in peak current. By application of the scheme illustrated in Figure 21 , an equally large (100 percent) frequency modulation was obtained (Figure 24).

\section{DISCUSSION}

The technological base on which the new devices rest is the coupling and pressure transformation technique which allows the tunnel diode to be compressed or tensed in a tightly integrated structure, without precarious alignment and displacement problems, without mechanical preload requirements, and with practically standard specifications for the diode itself. In principle, this development has brought the feasibility and practicality of the TDT close to that of the tunnel diode itself.

The TDT poses circuitry problems unlike those of conventional strain gages, since the electrical characteristics are strongly non-linear. However, the electromechanical transfer function can be well linearized. In fact, by judicious choice of operational parameters the non-linearity can be put to advantage to achieve certain desirable functions.

The TDT can be operated in various modes. In the stabilized mode the output signals can be readily processed by more or less standard techniques; however, the stabilization is achieved at the expense of added power drain (equal to about half the tunnel diode power dissipation) and the requirement of inconveniently low voltages. In the valley current mode, careful distinction has to be made between the "normal" and "anomalous" effects. The latter appears to be ruled out as a device mechanism because of stability and noise problems. That the "anomalous" effect is, in fact, 


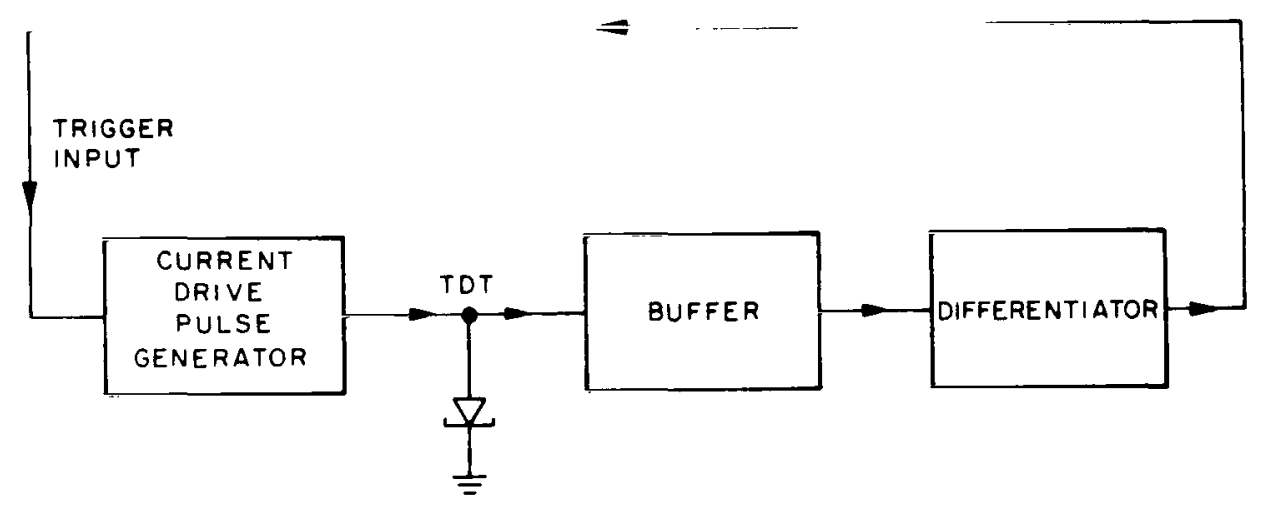

Figure 21.- Block diagram for a TDT frequency modulation scheme.

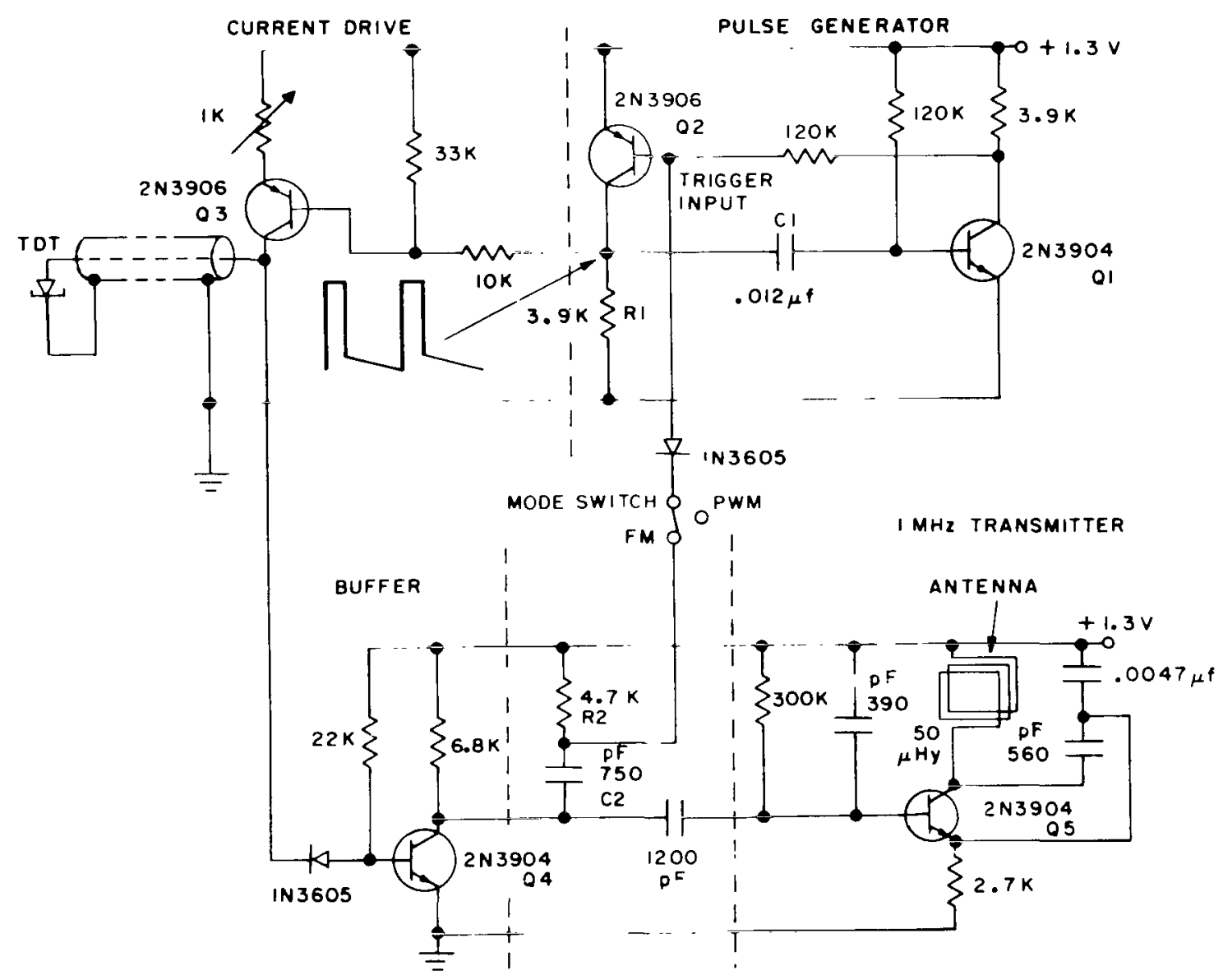

Figure 22.- Circuit for the TDT pulsewidth modulation and frequency modulation scheme and for a small 1-MHz transmitter. 


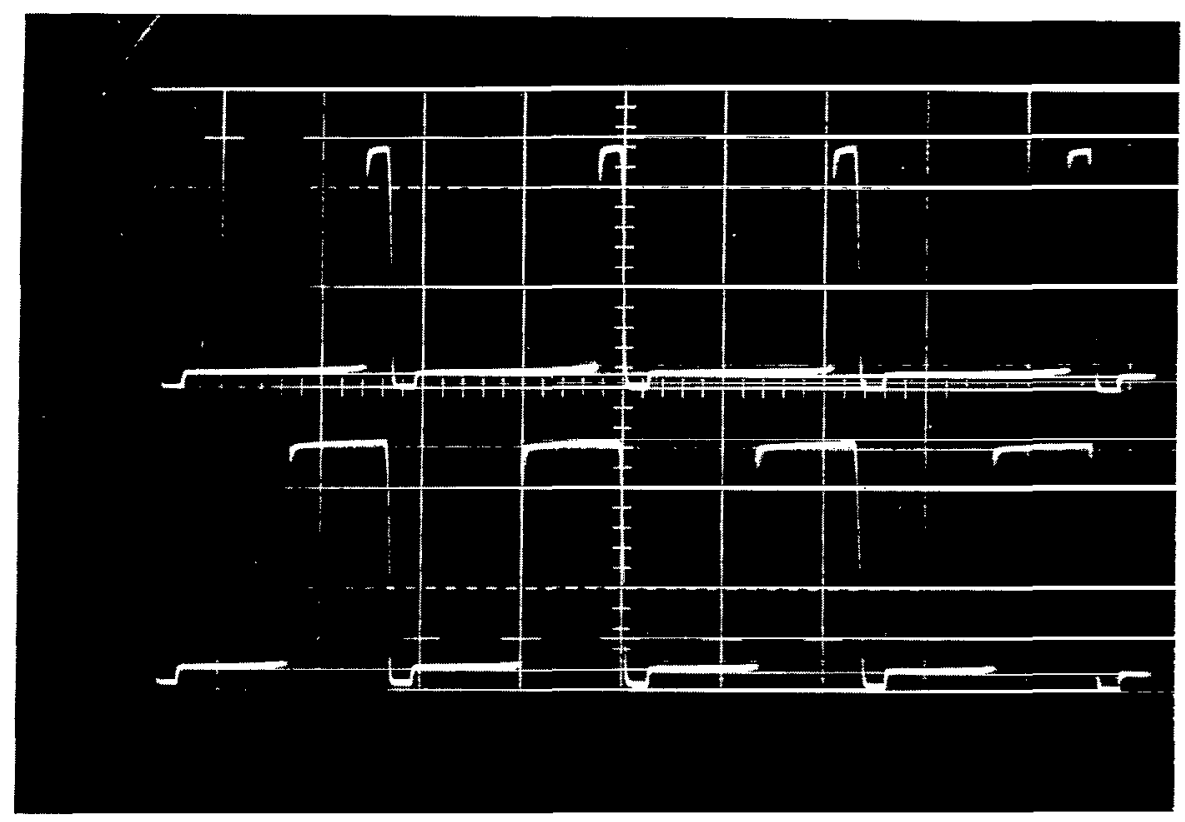

Figure 23. - Pulsewidth-modulated output of a TDT (time scale: $0.5 \mathrm{msec} / \mathrm{div})$, top: zero pressure, bottom: $150 \mathrm{~mm} \mathrm{Hg}$

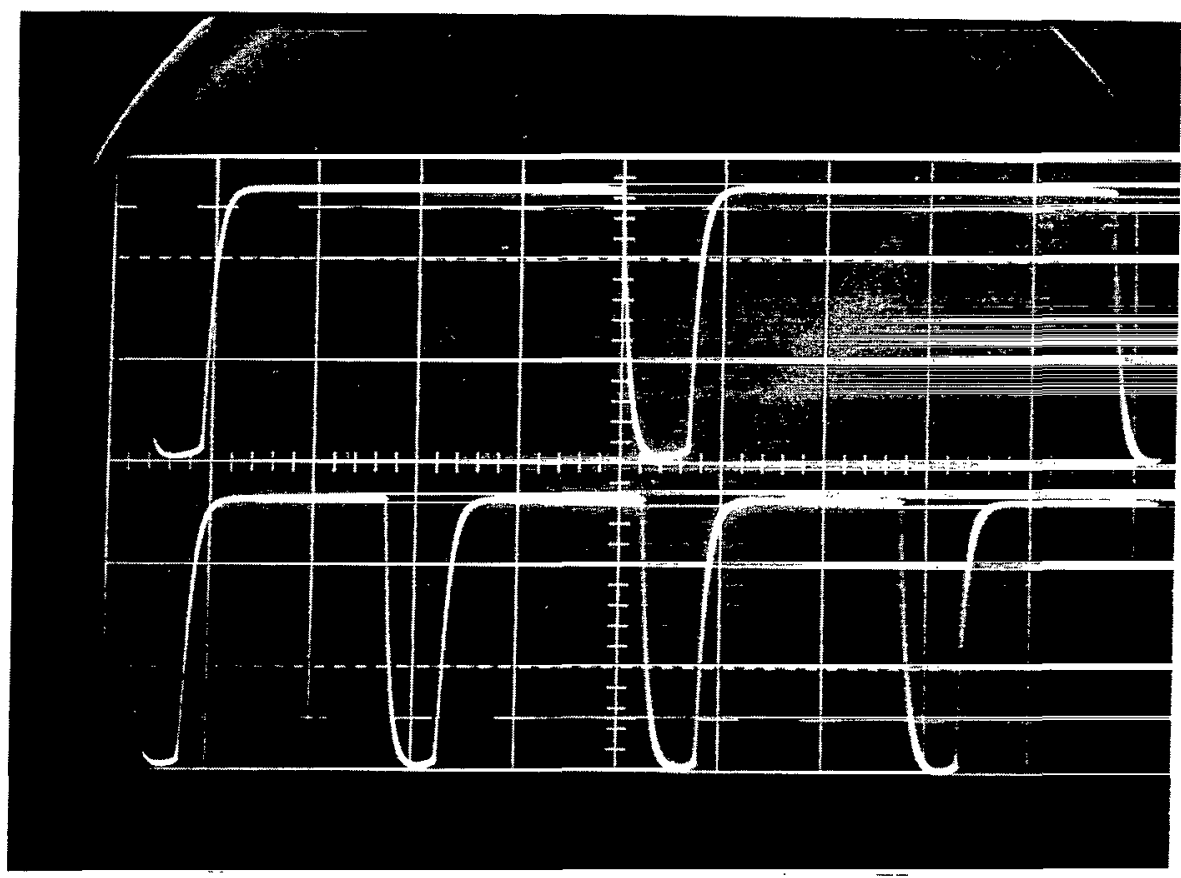

Figure 24. - Frequency-modulated output of a TDT (time scale: $0.2 \mathrm{msec} / \mathrm{div})$, top: zero pressure, bottom: $150 \mathrm{~mm} \mathrm{Hg}$ 
unsuited to device application is most readily apparent from the observation that a "normal" device can easily be made "anomalous" by overstressing. The precise nature of the mechanism underlying this "anomaly" is still unclear. Our present model, which involves the activation of indirect tunneling levels, certainly requires further investigation.

The "normal" valley current mode, which fits comfortably into a conventional band-gap model, offers interesting device capabilities; the required bias voltages match quite closely those of conventional energy cells, and the quiescent current and power levels are low. Furthermore, the bias range corresponding to very high differential resistance is quite large and no stabilization is required. However, under the usual condition of a large load resistance, provision must be made to switch the device to its operating bias point in the valley, and to reswitch to this point if a signal or instability causes a shift away from it. More seriously, this mode yields an inherently lower signal-to-noise ratio, since in the valley the low frequency noise is higher (ref. 18) and the signal current lower (ref. 13) than at the peak. Still this mode of operation is perfectly feasible, and may be desirable for certain applications.

Two more modes should receive at least passing mention: TDT's can be operated under reverse bias (ref. 9) with the advantage that no stabilization is required and the disadvantage that the low impedances involved ( 15 ohms) necessitate the use of amplifiers with very low equivalent input noise voltages. Iastly, of course, the device can be operated in the diffusion-current range under high forward bias, but under these conditions the TDT does not offer advantages over the piezodiode or piezotransistor.

One of the attractive features of the TDT is its small size, and the question naturally arises whether there is room for further miniaturization (without sacrifice of sensitivity). An attempt might be made to reduce the critical diaphragm size while compensating for the consequent loss of sensitivity by a more efficient transfer of mechanical energy. Reference to Figures 2 , 6 , and 10, which show, respectively, the pressure dependence of Gasb tunnel diodes, the junction diameter and the TDT pressure response suggests, however, that only limited size reduction can be achieved in this way. The junction area is about $10^{-6} \mathrm{~cm}^{2}$; a pressure of $150 \mathrm{~mm}$ Hg over a nominal diaphragm area of about $1 \mathrm{~mm}^{2}$ corresponds to a pressure of about $2000 \mathrm{~kg} / \mathrm{cm}^{2}$ at the junction. According to Figure 2 this pressure should yield $\Delta I / I=0.3$, whereas Figure 10 shows 0.05 only (which corresponds to an actual pressure of $275 \mathrm{~kg} / \mathrm{cm}^{2}$ ). Hence, we have exploited about 15 percent of the ideally obtainable sensitivity for the given geometry. While this percentage might appear small, it probably can- 
not be increased realistically by more than a factor of 2 or 3; hence only limited size reduction can be achieved through improvement of mechanical efficiency.

There are other avenues open for further miniaturization; for example, the whole device can be scaled down. For this case the sensitivity $\Delta I / I / \Delta \sigma$, the $S / N$ ratio, and hence the resolution and dynamic range, will remain constant (while power consumption will be reduced) if the pressure transformation remains unchanged and the noise current in the $1 / f$ range is proportional to the device current (ref. 22).

Another avenue for improvement, presently being explored, is to use materials such as InSb and InAs whose band-gaps are far more pressure-sensitive (about 5 and 1.5 times, respectively) than that of GaSb. A disadvantage of this approach is that these materials have smaller band-gaps and hence are more temperaturesensitive.

Sensitivity and miniaturization are intimately tied to dynamic range. For the present configuration, the highest pressures we could apply non-destructively were of the order of $3000 \mathrm{~mm} \mathrm{Hg}$. On the basis of a bulk modulus of $5.8 \times 10^{5} \mathrm{~kg} / \mathrm{cm}^{2}$ (ref. 23) $\mathrm{such}$ a pressure input results in about 4500 microstrain at the junction, which is also the approximate upper operational limit of conventional semiconductor gages. At the lower end of the dynamic range the device performance is limited by $1 / f$ noise. Whether any improvements are possible here is open to question, since the low frequency noise mechanisms are not too well understood. It would appear, therefore, that the dynamic range observed $(\sim 60 \mathrm{~dB})$ is unlikely to be widened significantly.

The operational range can, however, be shifted towards very much higher pressures. In the present device, it is the large pressure transformation which places the operational range in the $\mathrm{mm} \mathrm{Hg}$ pressure region. Without transformation, using the hydrostatic approach of Sikorski (ref. 3) and others, (ref. 2l) the operational range lies in the $1000^{\prime} \mathrm{s}$ of $\mathrm{kg} / \mathrm{cm}^{2}$ (and the tunnel diode requires essentially no mechanical adaptation to act as a transducer).

A very important device characteristic is the temperature-dependence. Comparison of the temperature-sensitivity of an original tunnel diode with that of the completed TDT established that the temperature effects are associated primarily with the junction itself. It will be recalled that the temperature-dependence of tunnel diodes is relatively small compared with that of non-degenerate junctions; a crude comparison of a Gasb tunnel diode with a forward-biased non-degenerate diode shows, in fact, an order of magnitude difference. Nevertheless, considering the pressure 
sensitivity of the TDT, the temperature effects are far from negligible. The complexity of the problem is apparent from Figures 12 and 13: not only does the baseline shift with temperature but the sensitivity also is temperature-dependent. The solution can take several forms: the simplest is to use tunnel diodes with appropriate doping levels. Recent work by Kovalev and Logunov (ref. 16) in GaSb has shown that a reduction of temperaturedependence is possible ( $I_{p}$ variations of less than 10 percent over the range $-100^{\circ} \mathrm{C}$ to $+100^{\circ} \mathrm{C}$ and less than 2 percent from 0 to $+50^{\circ} \mathrm{C}$ ) representing an improvement by more than an order of magnitude over our present tunnel diodes. A more elaborate scheme based on controlled doping would involve combining two diodes with temperature coefficients of opposite sign. Finally, the conventional approaches of using bridge and thermistor circuits (the latter effectively simulated by the conditions indicated in Figure 14) are readily adaptable to the TDT. How effectively even a simple conventional compensation scheme can be applied to reduce the temperature dependence by one or two orders of magnitude is well illustrated in Figure 19. For operation in narrow temperature ranges, such as encountered in certain biomedical applications, the temperature performance even of uncompensated TDT's is within quite acceptable limits. 


\section{REFERENCES}

1. Esaki, L.: New Phenomenon in Narrow Germanium $p-n$ Junctions. Phys. Rev., vol. 109, p. 603, 1958.

2. Esaki, I., and Miyahara, Y.: A New Device Using the Tunneling Process in Narrow $\mathrm{p}-\mathrm{n}$ Junctions. Solid-State Electronics, vol. $1, \mathrm{p} .13,1960$.

3. Sikorski, M.E.: Sensitive Tunnel Diode Pressure Transducers. ISCC Conf. Paper, vol. TA 7.5, 1962.

4. Rogers, E.S.: Experimental Tunnel Diode Electromechanical Transducer Elements. Soc. Am., vol. 34, p. 883, 1962 .

5. Kiggins, T.R., and Milnes, A.G.: A Solid-State Tunnel Diode Strain Gage. ISA Paper, 43.3.63, 1963.

6. Rindner, W., and Garfein, A.: Miniature Tunnel Diode Transducer. Solid-state Electronics, vol. 10, p. 1227, 1967.

7. Rindner, W., and Garfein, A.: A Miniature Piezo-Junction Pressure Transaucer. Proc. IEEE, vol. 56, p. 1129, 1968 .

8. Rindner, W., Doering, G., and Wonson, R.: Structural and Operational Characteristics of Piezo-Transisitors and Allied Devices. Solid-State Electronics, vol. 8, p. 227,1965 .

9. Sikorski, M.E.: Physical Acoustics. Vol. 1, part B, W.P. Mason, Ed., Academic Press, 1964.

10. Kane, E.O.: Theory of Tunneling. J. Appl. Phys., vol. $32, \mathrm{p} .83,1961$.

11a. Pittelli, E., and Rindner, W.:Tunnel and Excess Currents in Stressed Esaki Diodes. Solid-State Electronics, vol. 10, p. 911,1967 .

1lb. Bernard, W., Rindner, W., and Roth, H.: Anistropic Stress Effect on the Excess Current in Tunnel Diodes. J. Appl. Phys., vol. 35, p. 1860,1964 .

12. Nathan, M.I., and Paul, W.: The Pressure Dependence of Tunneling in Esaki Diodes. Int. Conf. Semiconductor Physics, p. 209, Prague, 1960.

13. Claassen, R.S.: Excess and Hump Current in Esaki Diodes. J. Appl. Phys., vol. 32, p. 2372, 1961. 


\section{REFERENCES (Cont.)}

14. Sladek, R.J.: Effect of Stress on the Electrical Properties of n-type GaAs. Phys. Rev., vol. 140, p. A1345, 1965.

15. Anashkina, T.N., et al.: Influence of Uniform Pressure and of Temperature on the Characteristics of $p-n$ Tunnel Junctions in GaAs. Sov. Phys. Solid-state, vol. 9, p. 219, 1967 .

16. Kovalev, A.N., and Logunov, L.A.: Gallium Antimonide Tunnel Diodes. Radio Eng. and Electronic Physics, vol. 11, p. 1329,1966 .

17. Meyerhofer, D., Brown, G.A., and Sommers, H.S.: Degenerate Germanium I. Tunnel, Excess and Thermal Current in Tunnel Diodes. Phys. Rev., vol. 126, p. 1329, 1962.

18. Giblin, R.A.: Noise Spectrum Measurements on Tunnel Diodes. Electronic Eng., vol. 36, p. 766, 1964 .

19. Noble, F.W.: A Hydraulic Pressure Generator. J. Lab. Clinical Medicine, vol. 54, p. 897, 1959.

20. Polanyi, M.L.: Medical Applications of Fiber Optics. 6th Int. Conf. Medical Elect. and Biological Eng., p. 36-2, Tokyo, 1965.

21. Yerman, A.J.: The Tunnel Diode as an FM Hydrostatic Pressure Sensor. ASME Meeting, Paper, 63-WA-264, 1963.

22. Korablev, I.V., Potemkin, V.V., and Yunosov, F.: Low Frequency Noise in Backward Diodes. Radio Eng. and Electronic Physics, vol. 13, p. 605, 1968.

23. Bateman, T.B., McSkimini, H., and Whelan, J.H.: Elastic Moduli of Single Crystal Gallium Arsenide, J. Appl. Phys., vol. 30, p. 544, 1959. 


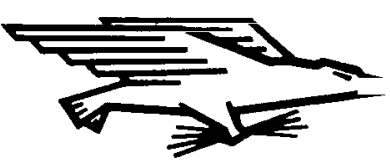

POSTAGE AND FEES PAID NATIONAL AERONAUTICS AN SPACE ADMINISTRATION

$03000134513 i 5 \quad 6932100903$

AR LURCF WEAPCNS LABCRATORY/WLIL/

KIRTLANO AIR FURCE BASE, NEW WEICU CIL1\%

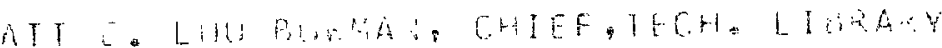

POSTMASTER :

If Undeliverable (Secrion 158 Postal Manual) Do Nor Rerurn

"The aeronatitical and space activities of the United States shall be conducted so as to contribute ... to the expansion of buman knowledge of phenomena in the atmospbere and space. The Administration shall provide for the widest practicable and appropriate dissemination of information concerning its activities and the results thereof."

\section{NASA SCIENTIFIC AND TECHNICAL PUBLICATIONS}

TECHNICAL REPORTS: Scientific and technical information considered important, complete, and a lasting contribution to existing knowledge.

TECHNICAL NOTES: Information less broad in scope but nevertheless of importance as a contribution to existing knowledge.

TECHNICAL MEMORANDUMS: Information receiving limited distribution because of preliminary data, security classification, or other reasons.

CONTRACTOR REPORTS: Scientific and technical information generated under a NASA contract or grant and considered an important contribution to existing knowledge.
TECHNICAL TRANSLATIONS: Information published in a foreign language considered to merit NASA distribution in English.

SPECIAL PUBLICATIONS: Information derived from or of value to NASA activities. Publications include conference proceedings, monographs, data compilations, handbooks, sourcebooks, and special bibliographies.

TECHNOLOGY UTILIZATION PUBLICATIONS: Information on technology used by NASA that may be of particular interest in commercial and other non-aerospace applications. Publications include Tech Briefs, Tcchnology Utilization Reports and Notes, and Technology Surveys.

Details on the availability of these publications may be obtained from:

SCIENTIFIC AND TECHNICAL INFORMATION DIVISION

NATIONAL AERONAUTICS AND SPACE ADMINISTRATION

Washington, D.C. 20546 